\title{
Intervention AUVs: The Next Challenge ${ }^{\star}$
}

\author{
Pere Ridao* Marc Carreras* David Ribas* Pedro J. Sanz ** \\ Gabriel Oliver ${ }^{* * *}$ \\ * Computer Vision and Robotics Research Institute \\ Scientific and Technological Park of the University of Girona \\ CIRS Building C/ Pic de Peguera, CO 17071 Girona \\ Catalonia - Spain (e-mail: \{pere,marcc,dribas\}@eia.udg.edu). \\ ** Universitat Jaume I (UJI), Av. Vicent Sos Banyat S/N, 12006 \\ Castelln, Spain (sanzp@uji.es) \\ *** Universitat de les Illes Balears (UIB), Cra. Valldemossa Km 7.5, \\ 07122 Palma, Spain (goliver@uib.es)
}

\begin{abstract}
While commercially available AUVs are routinely used in survey missions, a new set of applications exist which clearly demand intervention capabilities. The maintenance of: permanent underwater observatories, submerged oil wells, cabled sensor networks, pipes and the deployment and recovery of benthic stations are a few of them. These tasks are addressed nowadays using manned submersibles or work-class ROVs, equipped with teleoperated arms under human supervision. Although researchers have recently opened the door to future I-AUVs, a long path is still necessary to achieve autonomous underwater interventions. This paper reviews the evolution timeline in autonomous underwater intervention systems. Milestone projects in the state of the art are reviewed, highlighting their principal contributions to the field. To the best of the authors knowledge, only three vehicles have demonstrated some autonomous intervention capabilities so far: ALIVE, SAUVIM and GIRONA 500, being the last one the lightest one. In this paper GIRONA 500 I-AUV is presented and its software architecture discussed. Recent results in different scenarios are reported: 1) Valve turning and connector plugging/unplugging while docked to a subsea panel, 2) Free floating valve turning using learning by demonstration, and 3) Multipurpose free-floating object recovery. The paper ends discussing the lessons learned so far.
\end{abstract}

Keywords: Autonomous Vehicles, Robotic Manipulators, Marine Systems

\section{INTRODUCTION}

Nowadays, a relevant number of field operation in applications like marine rescue, marine science and the offshore industries, to mention some but a few, need intervention capabilities in order to perform the desired task. In such scenarios, most of the intervention operations are being undertaken by manned submersibles or by Remotely Operated Vehicles (ROVs), both equipped with robotic arms. Manned submersibles have the advantage of placing the operator in the field with direct view to the object being manipulated. Their drawbacks are the reduced time for operation (typically few hours), the human presence in a dangerous and hostile environment, and a very high cost associated with the need of an expensive oceanographic vessel to operate. Work class ROVs, are probably the more standard technology for deep intervention. They can be remotely operated for days without problems. Nevertheless, they still need an expensive oceanographic vessel with a heavy crane, an automatic Tether Management System (TMS) and a Dynamic Position system (DP).

* This work was supported by the Spanish project MERBOTS (DPI2014-57746-C3) and three European Commissions Sev- enth Framework Program 2011-2015 projects: FP7-ICT-2011-7- 288704 (MORPH) and FP7- INFRASTRUCTURES-2012-312762 (EUROFLEETS2).
Another issue is the cognitive fatigue of the ROV's pilot who has to take care of the umbilical and the ROV while coordinating with the operator in charge of the robotic arms. For all these reasons, some researchers have started to think about the natural evolution of the intervention ROV, the Intervention Autonomous Underwater Vehicle (I-AUV). Without the need for the TMS and the DP, light I-AUVs could be operated from vessels of opportunity reducing considerably the cost.

This paper surveys the principal achievements of the scientific community during the last 20 years of research and development in the field of autonomous underwater vehicles for intervention. First, relevant application domains for such technology are presented. Next, in section 3 , the most important projects undertaken in this area are reviewed pointing out their main contributions. In section 4, the recently developed GIRONA 500 I-AUV is presented, and its software architecture is then reported in section 5. A summary of the most relevant experimental results achieved so far with this I-AUV are described next in section 6. Finally, the main lessons learned are reported and some conclusions are drawn. 


\section{INTERVENTION APPLICATIONS}

\subsection{Oil and Gas Industry}

The oil \& gas industry is one of the principal users of underwater robotics technology. It uses work-class intervention ROVs to routinely inspect and repair the submerged infrastructures. AUVs have recently entered in this market, being already used to undertake geophysical surveys prior to pipe installation and, later on, for their inspection. The use of hovering type AUVs for the inspection of 3D infrastructures like submerged oil wells, chains, risers, etc. have started to be considered (Gilmour (2012)), although still in a research stage, since they represent major challenges for nowadays field capabilities. Few simple intervention tasks have also started to be considered by the research community. For instance, proof of concept demonstrations for tasks like acoustic-based homing to a sub-sea panel, real-time vision-based localisation relative to it, docking and opening a valve or plugging a hot stab have already been accomplished in simplified mockup environments.

\subsection{Rescue}

I-AUVs have a great potential in salvage operations. For instance, consider the search and recovery of the flight data recorder of a crashed plane. I-AUVs may contribute to the fast recovery of black-boxes since they can be easily mobilised using commercial airplanes world wide because of their small dimension and weight. Moreover, they can be launched from inexpensive ships of opportunity (non requiring DP) which are much more available than the expensive intervention/oceanographic vessels needed to deploy work-class intervention ROVs. After the localisation of the black-box with the help of advanced acoustic methods (like those reported in ACSA-ALCEN (2013)), these light I-AUVs could be deployed very fast, and taking profit of their high degree of automation, the recovery could be much more easily achieved at a reduced cost.

\subsection{Deep Water Archaeology}

During a significant part of the human history (one million years) the continental shelf was wider than nowadays. The sea level was about $130 \mathrm{~m}$ lower, and these coastal and lowland landscapes were attractive for human settlement (SPLASHCOS (2013)). About 16000 to 6000 years ago, after the last Ice Age, the sea level increased until current levels and these territories were drowned hiding important clues of our historical heritage. Shipwrecks, and in particular deep-sea wrecks (which may not be easily pillaged), are also a very important source of historical information. Underwater archaeologists are primarily interested in documenting submerged sites. High resolution 2D/3D seafloor mapping techniques are of high interest for them. There exist very few precedents of deep underwater excavations, mostly using high cost adhoc hardware or expensive ROV operations. However, most of the archaeological institutions are small, having at most access to small boats not equipped for deep intervention. Small and light HROVs (Hybrid Remotely Operated Vehicles) first, and I-AUVs later on, have a great potential to assist archaeologists beyond $50 \mathrm{~m}$ depth.

\subsection{Permanent Scientific Observatories}

Permanent observatories are artificial infrastructures located on the seabed which hold instrumentation which needs periodic maintenance. Common tasks include downloading vast amounts of data (for isolated non-cabled observatories), connecting/disconnecting an instrument, replacing batteries, instrumentation de-fouling, as well as placing and recovering sensor packages. I-AUVs have a direct application here, since they have the potential to be operated from inexpensive ships of opportunity drastically reducing the associated costs.

\section{STATE OF THE ART}

During the last 20 years, AUVs have become a standard tool for mapping the seafloor using optical (Eustice et al. (2006)) and acoustic (Paduan et al. (2009)) sensor modalities, with applications to infrastructure inspection (Ridao et al. (2010)), marine geology (Escartín et al. (2008)) and underwater archaeology (Bingham et al. (2010)) to name a few. After years of research, few autonomous platforms are already available in the market, most of them able to perform side scan sonar and bathymetric multi-beam surveys. Other functionalities, mostly related to optical mapping like 2D photo-mosaics, are not yet available through off-the-shelf applications although they have been extensively demonstrated in field application by several research institutions (Richmond and Rock (2007); Sigh et al. (2004); Ferrer et al. (2007)). 3D optical maps are nowadays one of the major fronts of research with some implementations already available based on monocular structure from motion (Pizarro et al. (2009); Nicosevici et al. (2009)), stereo (Johnson-Roberson et al. (2010)) and laser scanners (Inglis et al. (2012)).

However, a large number of applications exist which go beyond the survey capabilities. The maintenance of permanent observatories, submerged oil wells, cabled sensor networks, pipes, the deployment and recovery of benthic stations, or the search and recovery of black-boxes are just some of them. Nowadays, these tasks require the use of work-class ROVs deployed from DP vessels making them very expensive. To face these new applications, research to increase the autonomy of underwater intervention systems, started early in the 90s with the pioneering works with AUVs like OTTER (Wang et al. (1995)) and ODIN (Choi et al. (1994)), as well as projects like AMADEUS (Lane et al. (1997), fig.2) and UNION (Rigaud et al. (1998), fig.3), but it was not until the 1st decade of the 21th century that field demonstrations arrived. Very successful approaches where based on hybrid ROV/AUV concepts like the one proposed by the SWIMMER project (Evans et al. (2001), fig.4) where a shuttle AUV transporting an ROV, autonomously homes and docks into a seabed docking station. Next, the ROV, which is connected through the docking device to a remote operation station, is teleoperated during the intervention. The system avoids the need for a DP capable ship with the consequent savings.

Recently, another hybrid concept appeared, the HROVs (Fletcher et al. (2008)). These vehicles are essentially AUVs, reconfigurable as ROVs when tethered through an optical fiber umbilical. Thanks to its ultra light umbilical, 
HROVs may also be operated from ships of opportunity without DP. When plugged, HROVs behave as conventional ROVs avoiding some of the difficulties related to the cable. Moreover, they have the capability of detaching the cable before surfacing autonomously.

The most advanced demonstration up to date showed a wireless tele-operated intervention using light communications system from the HROV to an underwater gateway connected to an umbilical (Farr et al. (2010), fig.10).

However, both systems keep the human within the control loop. The first fully autonomous intervention at sea was demonstrated by the ALIVE project (Evans et al. (2003), fig.5), where a hovering capable AUV was able to home to a subsea intervention panel using an imaging sonar, and then, docking into it with hydraulic grasps using visual feedback. Once attached to the panel, a very simple manipulation strategy (fixed base manipulation) was used to open/close a valve. The first object manipulation from a floating vehicle (I-AUV) was achieved in 2009 within SAUVIM project (Marani et al. (2009), fig.6). It was demonstrated the capability of searching for an object whose position was roughly known a priori. The object was endowed with artificial landmarks and the robot autonomously located it and hooked it with a recovery device while hovering. Finally, the first multipurpose object search and recovery strategy was demonstrated in the TRIDENT project in 2012 (fig.7). First, the object was searched using a down-looking camera and photomosaicing techniques. Next, it was demonstrated how to autonomously "hook" the object in a water tank (Prats et al. (2011)). The experiment was repeated in a harbour environment using a 4 DOF arm (Prats et al. (2012b)), and later on with a 7 DOF arm endowed with a 3 fingered hand (Sanz et al. (2012), fig.1).

Nevertheless, according to (Gilmour (2012)) the technology for light intervention systems is still immature, but very promising. I-AUVs are currently in level 3 out of 9 (9 meaning routinely used) of the development cycle necessary to adopt this technology in the oil and gas industry, being expected to achieve up to level 7 by the end of 2018. I-AUVs will be necessary for efficient development of deep-water fields, in particular those where there are long subsea tie-backs with no surface facilities. They will be even more critical in future under ice activities with limited vertical access. Other application domains, like permanent scientific underwater observatories, share most of the same needs and problems (Drogou07 (2007)): Inspection and survey with video, performing manipulation tasks, docking to infrastructures and devices, sub-systems transport/deployment and handling surface-wire guided deployments/recoveries of devices. Plugging connectors, changing batteries, placing and recovering instruments are common activities, which would benefit from these autonomous manipulation skills.

In the next subsection the more representative projects involving autonomous intervention are reviewed in a chronological order, remarking their main contribution as well as the principal results achieved.

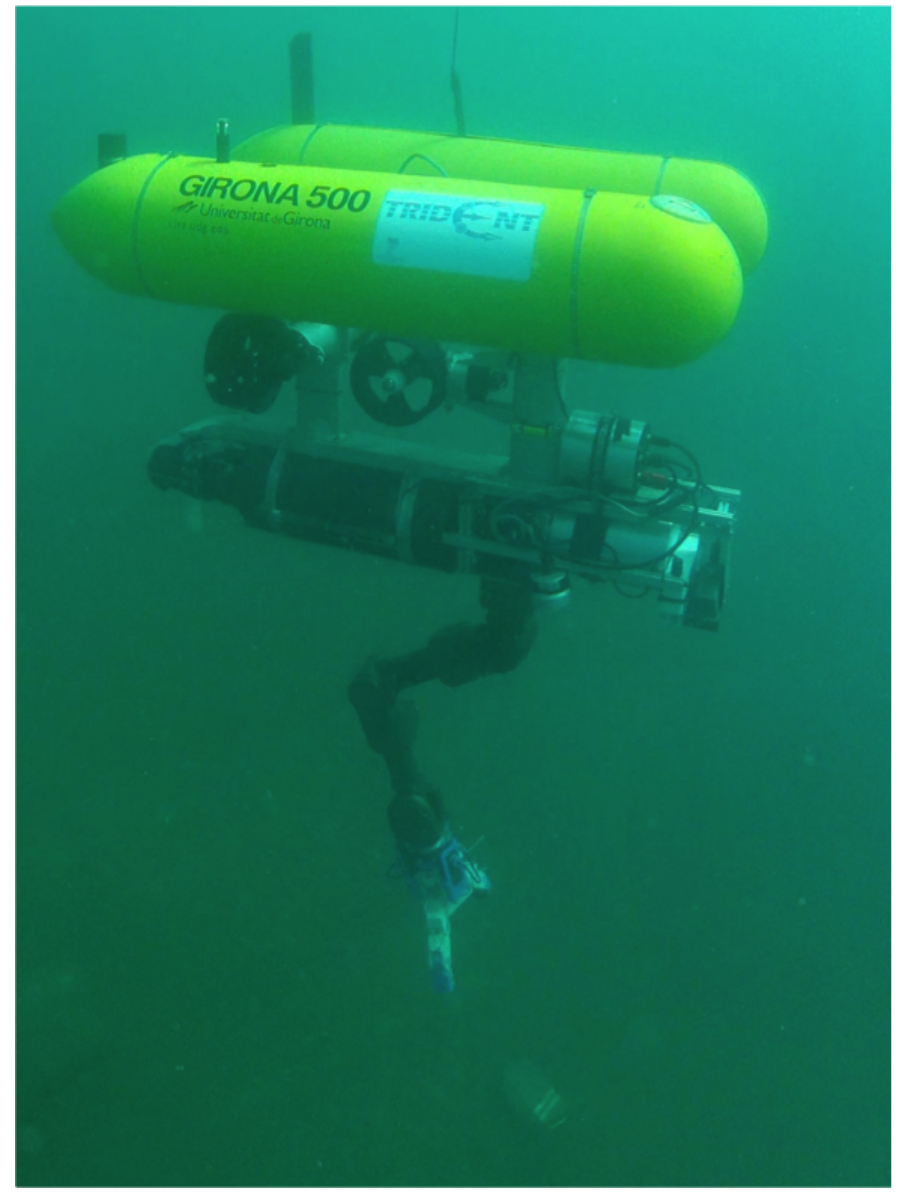

Fig. 1. GIRONA 500 in the TRIDENT I-AUV configuration.

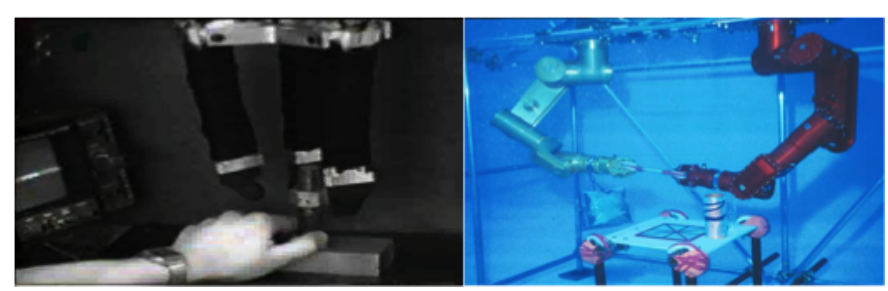

Fig. 2. AMADEUS concept

\subsection{Relevant Research Projects}

AMADEUS 1993-99 The project was divided in two phases. Phase I represents the first attempt to develop a dexterous gripper suitable for underwater applications (Lane et al. (1997)). The three fingered gripper was hydraulically actuated and coordinately controlled by mimicking, within each finger, the motions of an artificial elephant trunk. Phase II was devoted to the coordinated control of two underwater 7 DOF electro-mechanical arms (Casalino et al. (2002)). Each arm weighted $65 \mathrm{~kg}$, measured $140 \mathrm{~cm}$, and was filled with oil enabling it to reach a depth of $500 \mathrm{~m}$. To the best of the authors knowledge, AMADEUS represents the first demonstration underwater of an electrical 7 DOF arm with similar capabilities like those provided by of-the-shelf industrial manipulators. The project demonstrated the coordinated motion of the two end effectors while manipulating a rigid object inside a water tank. 

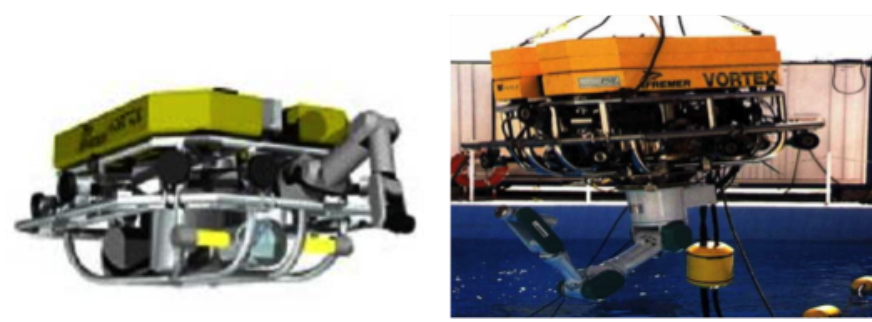

Fig. 3. UNION concept

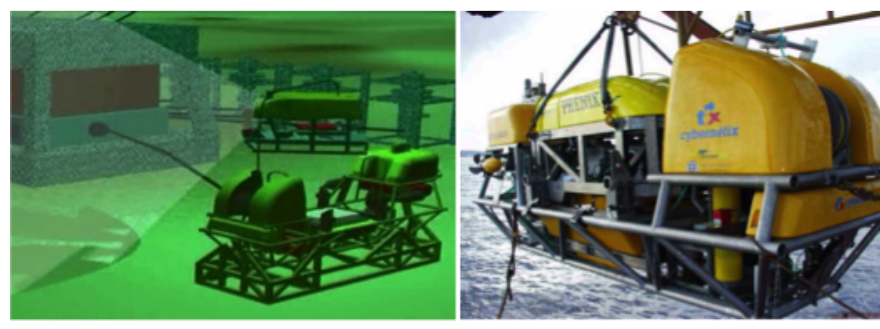

Fig. 4. SWIMMER concept

UNION 1996-99 This was a pioneering project with the aim to develop methods for increasing the autonomy and intelligence for ROVs (Rigaud et al. (1998)). The project focused mainly on the development of coordinated control and sensing strategies for combined manipulator (PA10) and vehicle (VORTEX) systems. The joint dynamics of the vehicle-manipulator system were studied and a robust non linear control proposed. To the best of the authors knowledge, UNION represents the first mechatronic assembly of a complete vehicle-manipulator system for automated manipulation. Nevertheless, the authors have failed to find published experimental manipulation results with the complete vehicle-manipulator.

SWIMMER 1999-01 It is a hybrid AUV/ROV intervention system conceived for the permanent inspection, maintenance, and reparation operations over deep water oil production facilities (Evans et al. (2001)). A ROV umbilical is integrated between the surface facility and the subsea site. The SWIMMER system is composed of an shuttle AUV which transports an intervention ROV to the subsea. SWIMMER is able to autonomously transit to the seafloor and docks to a subsea cradle-based docking station. The cradle is cabled to the teleoperation site. Once docked, the transported ROV is deployed and connected to the shuttle and through it to the docking station by means of an excursion umbilical. The intervention is carried out in a conventional teleoperated way. SWIMMER contribution consists on getting rid of the TMS and the expensive intervention vessel.

ALIVE 2001-04 ALIVE is a milestone project in autonomous underwater intervention (Evans et al. (2003)). The ALIVE 3.5 Ton vehicle was equipped with two hydraulic grasps for docking and a 7 DOF manipulation arm. It has been reported as the first AUV able to autonomously carry out a manipulation action consisting in opening/closing a valve in a subsea panel. ALIVE intervention concept is based on docking to a subsea panel to perform fixed-base manipulation. There is no interaction between the arm and the vehicle, and, hence, the manipulation becomes a conventional single arm manipulation but

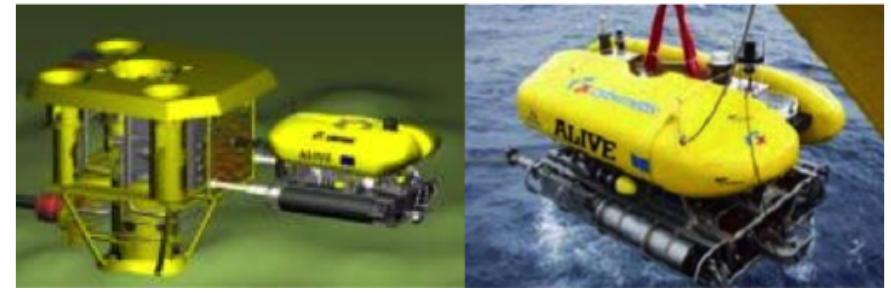

Fig. 5. ALIVE concept

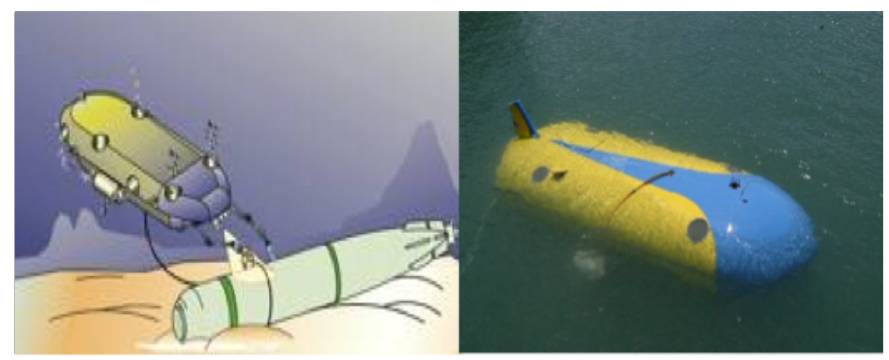

Fig. 6. SAUVIM concept

underwater. During the final demo of the ALIVE project the system proved its capability to autonomously navigate, dock and open a valve on an underwater panel similar to those of the oil industry.

SAUVIM 1997-09 SAUVIM is also a milestone project (Marani et al. (2009)). It was funded by the Office of Naval Research and was carried out at the Autonomous System Laboratory of the University of Hawaii. It was coexistent with ALIVE, but proposed a very different approach to autonomous intervention. SAUVIM focused on the free floating manipulation concept. The weight of the AUV (approx 6 Ton) was much larger than that of the arm (just $65 \mathrm{Kg}$ ), and therefore, both systems were considered as practically uncoupled. In other words, the perturbations produced on the AUV pose as result of the motion of the arm were negligible. Because of that uncoupled controllers were implemented. SAUVIM demonstrated accurate navigation and station keeping and was the first project to demonstrate the autonomous recovery of an a priori known object. It is worth noting that SAUVIM used the ANSALDO arm previously built in the context of the AMADEUS project.

RAUVI 2009-11 RAUVI was a Spanish funded project devoted to the design and implementation of a reconfigurable AUV for intervention missions. The major outcome of RAUVI was the development of the GIRONA 500 I-AUV (Ribas et al. (2012)) which was equipped with an ECA/CSIP electrically driven arm with 4 DOFs (Fernandez et al. (2013)). The AUV was equipped with a stereo pair and was used to demonstrate autonomous object recovery in a water tank environment (Prats et al. (2012d)). To the best of authors knowledge, after ALIVE and SAUVIM, RAUVI is the 3rd project which demonstrated experimentally autonomous intervention capabilities, being the one using the lightest vehicle (less than 200 $\mathrm{kg})$. RAUVI was the seed of a more ambitious EU project named TRIDENT.

TRIDENT 2010-12 This project proposed a new methodology for multipurpose underwater intervention tasks 


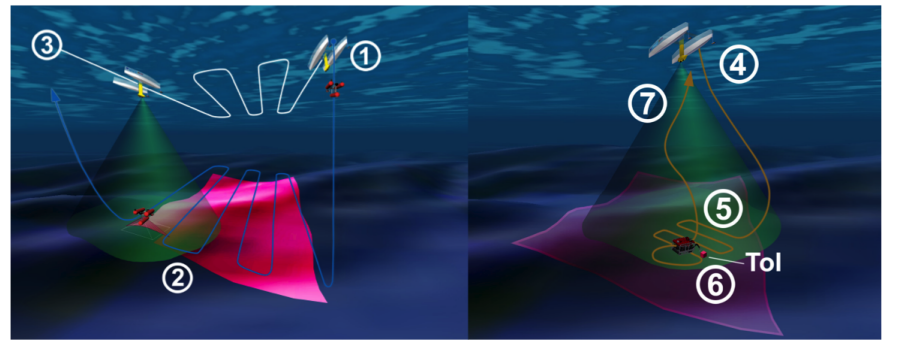

Fig. 7. TRIDENT concept

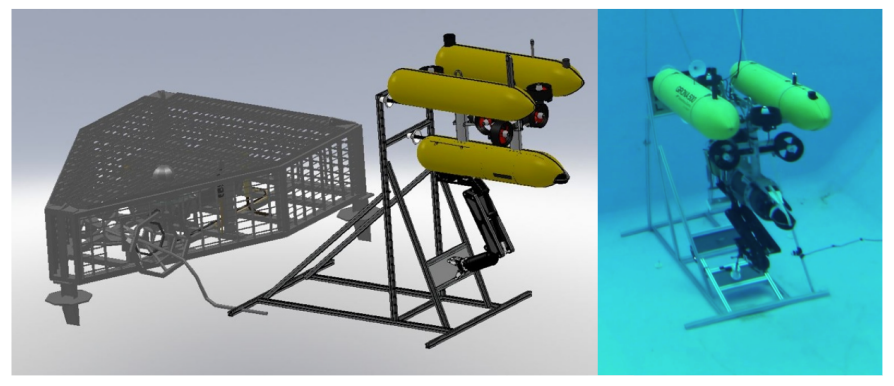

Fig. 8. TRITON concept

(Sanz et al. (2012)). A team of two cooperative heterogeneous robots with complementary skills, an ASC (Autonomous Surface Craft) and an I-AUV endowed with a dexterous 7 DOF manipulator and a 3-fingered hand, was used to perform underwater manipulation tasks. TRIDENT concept is divided on two phases: Survey and Intervention. During survey, the team of vehicles map the seafloor. Next, the I-AUV is recovered and a seafloor map is built. With the help of the map, the user selects an object and a desired intervention task. Then, the team of vehicles is deployed again to search for the target using the existing map as a reference. Once this is accomplished, the I-AUV performs a multisensory-based intervention through free-floating manipulation to recover the object. The TRIDENT concept has been demonstrated in a harbour environment in an uncoupled way: 1) The capability of both vehicles working in tandem during mapping and 2) the capability of the I-AUV to intervene over the target. As an evolution of RAUVI, TRIDENT became, together with ALIVE and SAUVIM a milestone project in autonomous underwater manipulation, providing for the first time field results in multipurpose object recovery.

TRITON 2012-14 Is a Spanish funded project which demonstrated autonomous docking to a custom subsea panel, fixed-based manipulation for valve turning and hot stab connection with application to the maintenance of permanent submerged observatories. Project demonstrations were carried out in a water tank (Palomeras et al. (2013)) as well as with sea trials in a harbour environment (Palomeras et al. (2014)).

PANDORA 2012-14 The main goal of PANDORA (Lane et al. (2012)) was to make autonomous robots persistently autonomous, reducing the frequency of assistance requests. The key of this objective is the ability to recognise failure and respond to it, at all levels of abstraction and time constants. The project developed three themes: (1) describing the world for detecting failures in the task execution; (2) directing and adapting intentions by means

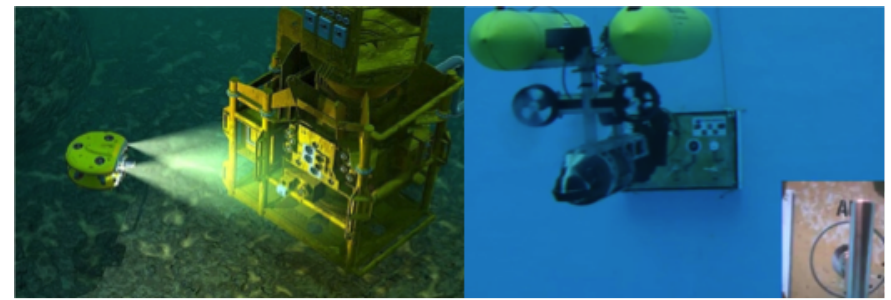

Fig. 9. PANDORA concept
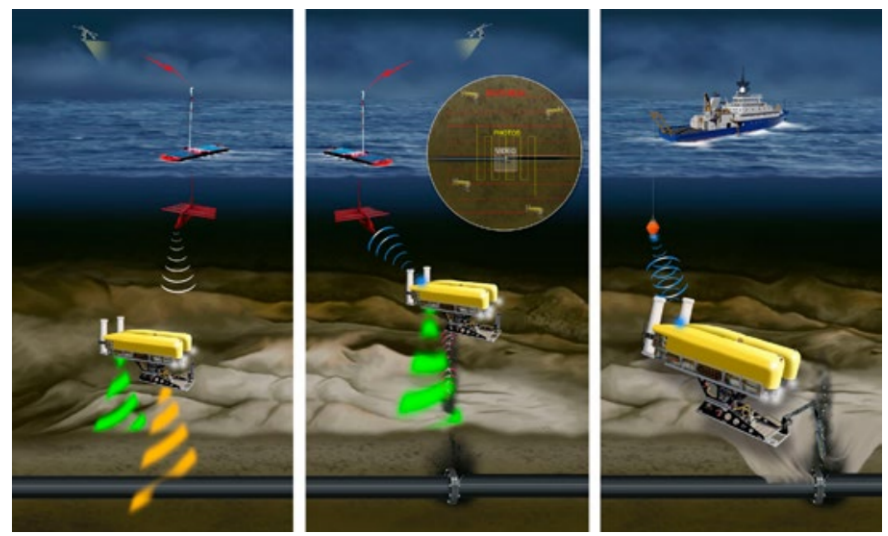

Fig. 10. Hybrid AUV inspection, monitoring, and intervention of seafloor and sub-seafloor pipelines concept.

of planning for responding to failures; and (3) acting robustly mixing learning and robust control for making actions indifferent to perturbations and uncertainty. The project centered its validation tasks on AUVs acting in an oil field scenario in which the robots perform inspection, cleaning and valve turning.

Hybrid AUV inspection, monitoring, and intervention of seafloor and sub-seafloor pipelines This very recent project (Kaiser et al. (2013)) proposes the cooperation of a surface vehicle and an underwater one. In this case a wave glider is used in surface for vehicle tracking and communications gateway. An HROV, working in AUV mode, is used to perform an autonomous survey close to the seafloor, for example, too look for leakages in buried pipes. If an anomaly is detected, the underwater vehicle performs a detailed survey of the area while waiting for the assistance of a surface support vessel. By lowering an optical communications package, the surveyed data is downloaded and the vehicle is switched to ROV mode to perform a light intervention while a work-class ROV is brought to the site for heavier work. Although this is a very recent project, wireless intervention for the teleoperation of NEREUS HROV through a high bandwidth opto/acoustic has been already demonstrated (Farr et al. (2010)).

\section{GIRONA 500 I-AUV}

The GIRONA 500 (Ribas et al. (2012)) is a compact-size AUV designed and developed in the University of Girona for a maximum operating depth of $500 \mathrm{~m}$. The vehicle is built around an aluminum frame which supports three torpedo-shaped hulls as well as other elements like the thrusters. The overall dimensions of the vehicle are $1 \mathrm{~m}$ in height, $1 \mathrm{~m}$ in width, $1.5 \mathrm{~m}$ in length and a weight (on its 

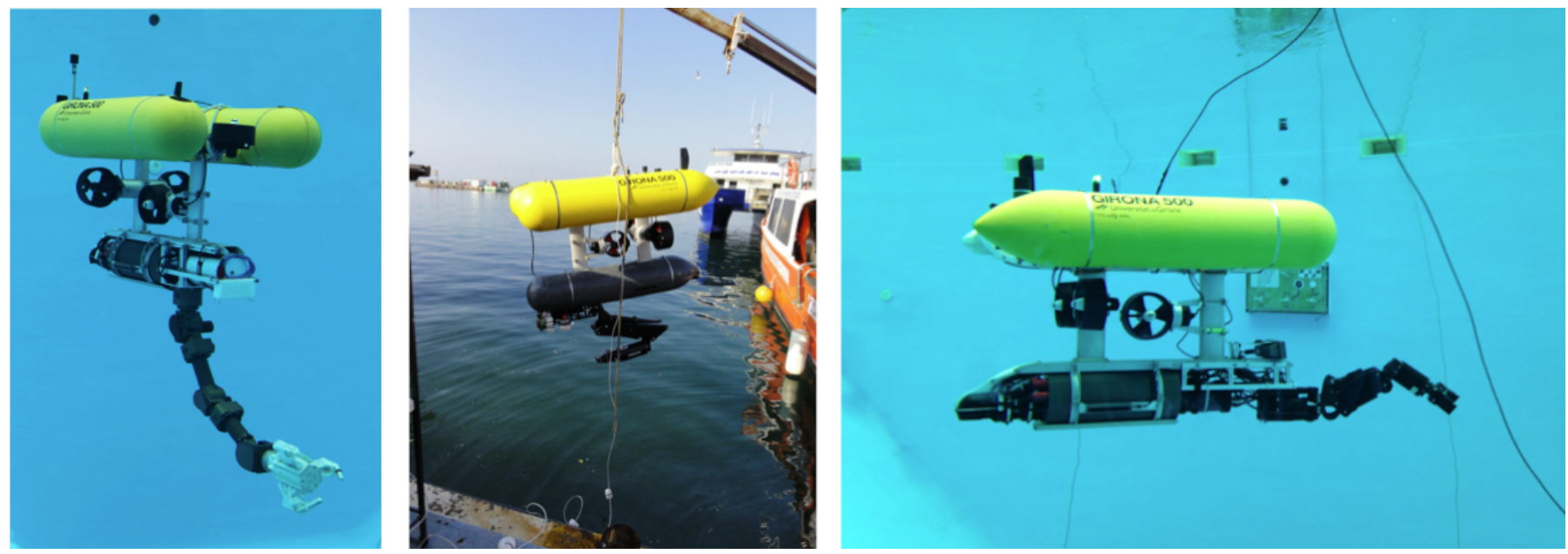

Fig. 11. GIRONA 500 I-AUV in: a) TRIDENT configuration with the GRAALTECH 7 DOF arm and the 3 fingered hand developed by University of Bolonia; b) RAUVI/TRITON configuration with the ECA/CSIP Light Weight 4 DOF arm; c) PANDORA configuration with the ECA/CSIP Micro 4 DOF arm.

basic configuration) of about $140 \mathrm{~kg}$. The two upper hulls, which contain the flotation foam and the electronics housing, are positively buoyant, while the lower one contains the more heavy elements such as the batteries and the payload. This particular arrangement of the components provides the vehicle with passive stability in pitch and roll, making it suitable for tasks requiring a stable platform such as video surveying or intervention.

The most remarkable characteristic of the GIRONA 500 is its capacity to reconfigure for different tasks. On its basic configuration, the vehicle is equipped with typical navigation sensors (DVL, AHRS, pressure gauge and USBL) and a basic survey equipment (profiler sonar, side scan sonar, video camera and sound velocity sensor). In addition to these sensors, almost half the volume of the lower hull is reserved for mission-specific payload, which makes possible to modify its sensing and actuation capabilities as required. A similar philosophy has been applied to the propulsion system which can be set to operate with a different number of thrusters, ranging from 3 to 8 , to actuate the necessary degrees of freedom and provide, if required, some degree of redundancy.

In the context of autonomous intervention, three different payloads have been developed. The first one (fig.11b) was developed in the context of the RAUVI Spanish project and is composed of a light duty 4 DOF electrical manipulator, a video system and their corresponding control electronics. The main goal of the project was to perform a two-step autonomous underwater intervention mission consisting of an initial video survey phase in which a particular object was localised, and then retrieved using a hook attached to the robotic arm. This same configuration has been later used in the TRITON Spanish project to demonstrate more challenging tasks, such as the manipulation of valves and connectors, while docked at an intervention panel using a simple gripper as end effector. The second payload (fig.11-a) was developed as part of the TRIDENT FP7 project. The main difference with the previous one is the higher level of dexterity of the system achieved with a 7 DOF manipulator and a three-fingered hand. This made possible to demonstrate grasping capabilities for recovery tasks, while opening the door to the manipulation of objects with more complex shapes. Finally, the last payload (fig.11-c) was built for the PANDORA FP7 project. A new small size 4 DOF arm was integrated into the GIRONA 500 to demonstrate the autonomous free-floating operation of valves on an intervention panel. For that purpose, a fixed "V" shaped tool was installed as end effector to actuate the valves. The tool included a camera at the vertex of the fixed-jaw, looking forward in the direction of the approach vector, allowing to detect the valve orientation during the manipulation. The arm was mounted on the front part of the vehicle to provide a convenient workspace. Finally, a force-torque sensor was mounted on the wrist to measure the interaction force with the valve.

\section{COLA2: GIRONA 500'S SYSTEM ARCHITECTURE}

The System's Architecture of the GIRONA 500 I-AUV has three main components: one to control the AUV, another to control the arm and the last one is devoted to the coordination of the two previous components.

\subsection{AUV Software Architecture}

GIRONA 500 uses a software architecture that integrates perception, learning, action and communication. The architecture is composed of several modules which are organized in layers, see Fig. 12.

Sensing and Actuation Layer Starting from the top of Fig. 12, the first layer contains all the sensors and actuators drivers. It uses the Attitude Heading Reference System (AHRS) to measure the angular velocity $\left(\nu_{2}\right)$ and the robot attitude $\left(\eta_{2}\right)$, the Doppler Velocity Log (DVL) to measure the linear velocity $\left(\nu_{1}\right)$, the depth sensor included within the Sound Velocity Sensor device (SVS) is used to measure the depth $(z)$, the acoustic modem measures ranges against another acoustic modem acting as transponders $(r)$ and a stereo camera is used to gather stereo imagery $\left(I=\left\{I_{1}, I_{2}\right\}\right)$. This layer also includes the drivers for the thrusters which are controlled in speed. 


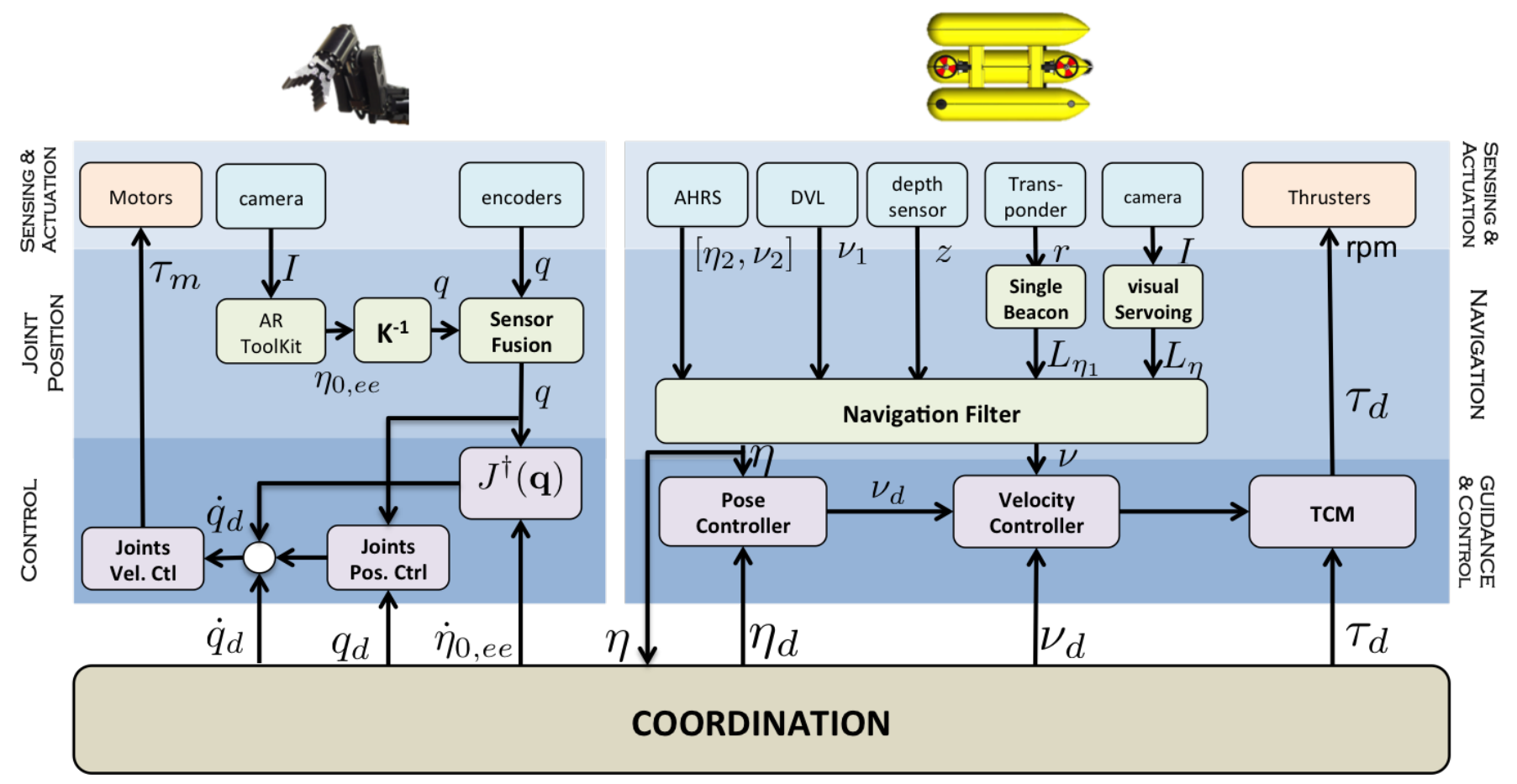

Fig. 12. GIRONA 500's System Architecture

Navigation Using a constant velocity model with attitude input $\left(\eta_{2}\right)$, and depth $(z)$ and linear velocity measurements $\left(\nu_{1}\right)$, an Extended Kalman Filter (EKF) is used to estimate the robot position and velocity $\left(\boldsymbol{x}_{\boldsymbol{k}}=\left[\eta_{1}^{T} \nu_{1}^{T}\right]^{T}\right)$ by dead reckoning. The single beacon module is used for single beacon navigation. It uses an active localization strategy making use of a Sum of Gaussian (SoG) filter (Vallicrosa et al. (2014)) to estimate the beacon position $\left(L_{\eta_{1}}\right)$. The visual servoing module (Palomeras et al. (2014)) uses as input the camera images $(I)$ as well as a template of an object of interest to estimate the object pose $\left(L_{\eta}=\left[\begin{array}{ll}L_{\eta_{1}}^{T} & L_{\eta_{2}}^{T}\end{array}\right]^{T}\right)$ with respect to the robot (using a position based visual servoing method). When doing intervention in subsea panels as described in section 6.1 a transponder is mounted on the subsea panel and the panel-template is used for visual servoing. In this case, the panel pose is incorporated in a Navigation Filter module which uses a single feature EKF SLAM (Simultaneous Localization and Mapping) method to simultaneously estimate the robot position and velocity and the beacon pose $\left(\boldsymbol{x}_{\boldsymbol{k}}=\left[\eta_{1}^{T} \nu_{1}^{T} L_{\eta_{1}}^{T} L_{\eta_{2}}^{T}\right]^{T}\right)$ to bound the navigation drift.

Guidance and Control The AUV control is based on a nested pose/velocity PID (Proportional-IntegralDerivative) controller. At the inner level, the velocity controller computes the force and torque $\left(\tau_{d}\right)$ to be applied to reach the desired velocity $\left(\nu_{d}\right)$. It is computed by combining a standard 4 DOF (surge, sway, heave and yaw DOFs) PID control with a feed-forward model which provides the nominal force to be applied to achieve a certain velocity. The output of the velocity controller $\left(\tau_{d}\right)$ is later allocated to the thrusters using the thruster allocation matrix within the Thurster Control Matrix module. Once the force to be exerted by each thruster is known, a static thruster model is used to convert from force into the thruster setpoint. In a hierarchical upper level, a pose controller is in charge of the AUV pose $(\eta)$ regulation and tracking. It works by sending velocity setpoints $\left(\nu_{d}\right)$ to the velocity controller and reading the pose feedback $(\eta)$ from the Navigation module, to achieve a desired pose $\left(\eta_{d}\right)$.

\subsection{Arm Software Architecture}

Similarly to the vehicle, the arm's control architecture is also structured in three layers:

Sensing and Actuation This layer gives access to the physical sensors and actuators of the arm. It includes the access to the motor drivers, the joint encoders and a camera used to estimate the end effector pose through visual servoing.

Joint Position Although arm position in configuration space is instrumented with joint encoders, some arms may lose pulses during operation which leads to a bad calibration. To deal with this problem, the end effector is labeled using a AR code (Kato and Billinghurst (1999)) whose pose can be easily estimated with a camera. Then, by means of inverse kinematics $\left(K^{-1}\right)$ it is possible to estimate the arm configuration $(Q)$ which is used to recalibrate (Sensor Fusion module) the one read from the encoders.

Joint Control A cascade joint position and velocity controller is used to control each robot arm joint. Hence it is possible to control the joint velocity and/or position. It is also possible to control the end effector velocity $\left(\dot{\eta}_{0, e e}\right)$ in Cartesian space by means of the arm pseudo inverse Jacobian $\left(J^{\dagger}(\mathbf{q})\right)$ which translates the Cartesian velocity into the joint velocity $(\dot{\boldsymbol{q}})$. 


\subsection{Coordination}

The coordination layer is devoted to the joint control of the AUV and the robot arm. Different coordination mechanisms have been implemented during the last years for the different intervention experiments performed. In Prats et al. (2012b), the AUV performs station keeping while the robot arm is controlled to grasp an object from the seafloor, using an uncoupled control of the AUV and the robot arm. The same happens in Palomeras et al. (2014), where the coordination is based on a state machine which sequences the AUV and arm operation. First the AUV docks into a docking station and then the arm is used to turn a valve as well as to plug/unplug a connector. In Carrera et al. (2014), a joint control of the AUV and the robot arm is used and the coordination mechanism is based on the proposed Learning by Demonstration method reported. Finally in Cieslak et al. (2015) and Sanz et al. (2012) a variation of the task priority redundancy control framework were used to control simultaneously the AUV and the arm to drive the end effector during the autonomous intervention task.

\section{EXPERIMENTS}

During the last years we have found very useful the definition of realistic mission scenarios to drive our research and development. Forcing ourselves to demonstrate the technology in realistic scenarios or mock-ups, roots our work to the real problems that must be solved to make autonomous intervention a reality. In order to allow for a smooth transition from simulation to experimental validation, the proposed scenarios are implemented at least at three levels of complexity: 1) Graphical simulation, 2) Water tank testing and 3) Sea trials. In all cases, the condition of the experimental setup are clearly defined and easy to reproduce by third parties in order to promote reproducible research. In this section, the experimental scenarios being used in our recent projects are described and the already available results highlighted.

\subsection{Subsea-Panel Docking and Fixed-Base Manipulation}

This is the most simple and easy scenario, but also the one more close to field applications nowadays.

Problem statement Given a subsea panel equipped with a funnel-based docking mechanism, and a visual featurerich textured panel to allow for real-time vision based localisation, the I-AUV has to start at a random position with the panel in the field of view (it is assumed to have reached the panel vicinity by acoustic means) and dock autonomously to the panel. Next, it has to be able to complete an autonomous valve turning and connector plug/unplug actions. The valve and the connector have been placed inside the manipulator working space. A custom made connector based on a hot stab has been designed with passive accommodation. To allow for an easy reproduction by third parties, the docking station (fig.16) is made with cheap aluminium profiles, a digital image has been printed and laid on the panels and the components (funnels, valve and connector) have been printed in 3D.
Arm initialisation and visual servoing Prior to the experiment, when the I-AUV is started, the arm must be initialised in order to know its zero position in the joint space. To this aim, each joint is moved to its mechanical limit where its zero is fixed. Later, the hall effect sensors located in the electrical motors are used to track the joint angles. Nevertheless, the uncertainty in the kinematics model and the non-linearities in the linear-to-circular transmission used to move the rotative joins by means of electrical driven pistons, are responsible for the inaccuracy of the Cartesian position of the end effector, in particular at the boundaries of the working space. To solve this issue, a visual servoing approach has been followed. An ARToolKit Marker (Kato and Billinghurst (1999)) has been placed in the jaw grip. Placing the arm in a known position, and locating the marker with the camera, allows to estimate the camera to robot base transformation $\left({ }^{b} M_{c}\right)$. Later, each time the marker is detected within the camera, its pose is measured and using the arm inverse kinematics, the robot position in the configuration space is updated. This approach mitigates the arm inaccuracies ensuring consistency between the arm and the valve and connector poses.

Panel detection Detection of the underwater panel is performed using vision, by comparing the images from the camera against an a priori known template of the panel. By detecting and matching unique features in the camera image and template, it is possible to detect the presence of the panel, as well as accurately estimate the position/orientation when a sufficient number of features are matched.

In this work, we choose the oriented FAST and rotated BRIEF(ORB) (Rublee et al. (2011)) feature extractor for its suitability to real-time applications. The ORB feature extractor relies on features from accelerated segment test (FAST) corner detection (Rosten and Drummond (2006)) to detect features, or keypoints, in the image. These are obvious features to detect in man-made structures and may be detected very quickly. Moreover, there is a descriptor (binary) vector of the keypoint based on binary robust independent elementary features (BRIEF) (Calonder et al. (2010)). This allows us to rapidly obtain the difference between descriptors and allows real-time matching of keypoints at higher image frame-rates when compared to the other commonly used feature extractors such as scale invariant feature transform (SIFT) (Lowe (2004)) and speeded-up robust features (SURF) (Bay et al. (2008)). Fig. 13 illustrates the matching between the panel tem-

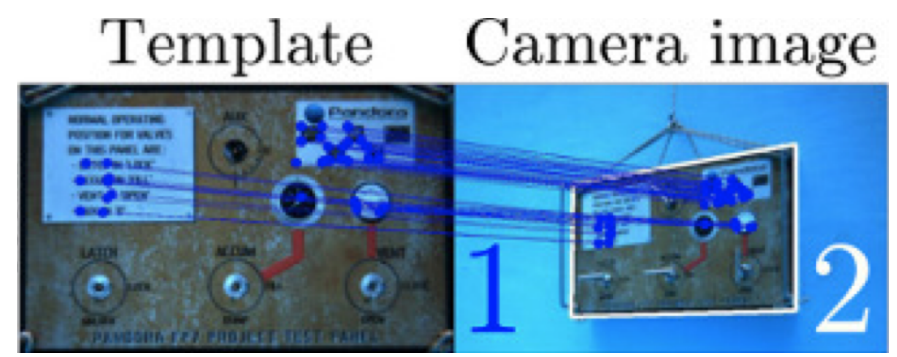

Fig. 13. Vision-based localisation with respect the panel.

plate and an image received from the camera. A minimum number of keypoints must be matched between the tem- 


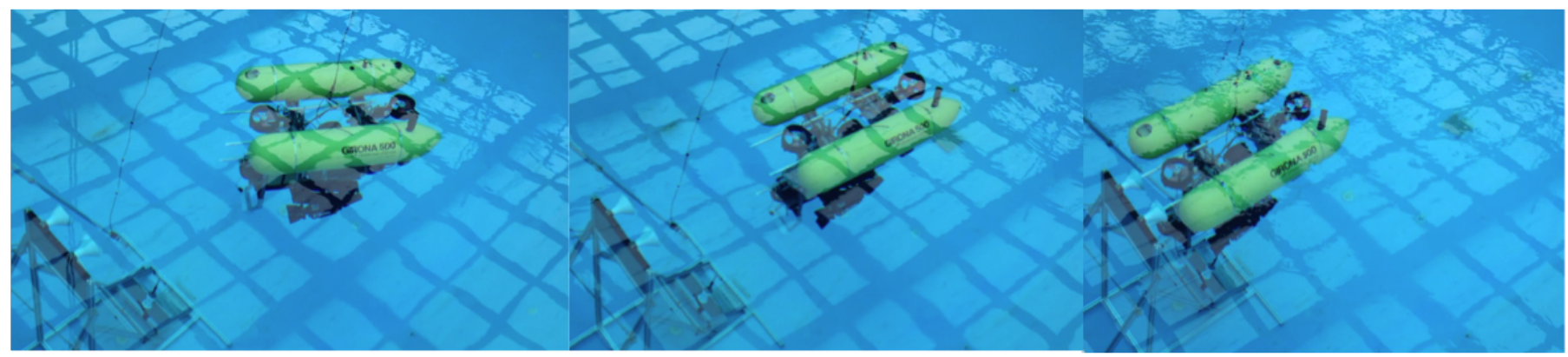

Fig. 14. Docking Sequence.

plate and the camera image to satisfy the panel detection requirement. A low number of matched keypoints indicates that the panel is not in the camera field of view. The correspondences between the template and camera image can be used to compute the transformation (or homography) of the template image to the detected panel in the camera image. This allows us to compute the image-coordinates of the corners of the panel in the camera image. Using the known geometry of the panel and the camera matrix, we are able to determine the pose of the panel in the camera coordinate system (Palomeras et al. (2013)).

Docking The vehicle starts in the vicinity of the panel with a visual contact already stablished. The above mentioned vision-based localisation algorithm is used to compute the panel position with respect to the robot. Next, a single landmark SLAM problem (Palomeras et al. (2013)) is instantiated so the robot and panel position become known in the world frame. With the panel mapped, vehicle localisation improves significantly due to visual feedback provided by the visual detector every time that the intervention panel is identified. The state machine governing the robot motion generates a waypoint just in front of the mapped panel position at a distance in which the intervention panel should be inside the vehicle field of view. This waypoint will be used later as a recovery position if next step fails. From this position the vehicle starts facing the intervention panel moving holonomically in 4 DOFs until the vehicle docking probes and the panel docking cones are aligned and separated approximately $0.25 \mathrm{~m}$. If during this process the visual detector is unable to detect the intervention panel, the facing step is aborted and the vehicle returns to the previous defined recovery waypoint. However, if as expected, the panel has been detected during the facing step, last step starts pushing the vehicle forward by sending directly a force in the $\mathrm{X}$-axis through the thruster allocator control node, while keeping the same depth and angle. Last step produces the mechanical coupling of both systems. Since no latching mechanism is used, to keep the AUV docked to the intervention panel it is necessary to keep pushing it with a desired force (i.e. $40 \mathrm{~N}$ with our vehicle) until the intervention operation is concluded. Few snapshots of the docking sequence are shown in fig. 14 .

Valve and Connector Detection For the valve/connector detection and operation, a stereo camera has been placed in the bottom hull of the GIRONA 500 I-AUV, pointing to the region where the object to be manipulated is supposed to be when the vehicle is already docked (see fig.15 top).
Regarding the target detection, two methods have been implemented that can run individual or simultaneously to increase its robustness (see fig. 15 bottom). First, a method that uses the histogram of hue and saturation in the HSV color space reported in (Prats et al. (2012d)), has been adapted to detect three red marks on the valve. Once the stereo correspondence of the marks is established, they are fitted to the tridimensional model of the valve using an optimal rigid transformation, obtaining the valve pose $\left({ }^{c} M_{o}\right)$ with respect to the camera. Alternatively, a landmark detection method has been also developed, not requiring stereoscopy. In that case two tags, one placed close to the connector base and the other on the gripper, are used. The detection of the tags allows a continuous visual feedback and provides the 3D transformation needed for the end effector to properly grip the connector handle. Both methods are based on the premise that the valve and the connector, as well as the supporting structure, are reasonably rigid and its geometry is known. New methods allowing some degree of uncertainty are under study for a more robust vision based manipulation approach.

Valve Turning and Connector Plugging/Unplugging After docking, the intervention begins. Two operations are performed: open/close a valve and plug/unplug a hot-stab connector. The main steps followed for the intervention are summarized hereinafter. Given an object to manipulate (valve or hot-stab) and given its camera relative pose $\left({ }^{c} M_{o}\right)$ provided by the above mentioned vision algorithm, the object's pose with respect to the arm-base can be easily computed as ${ }^{b} M_{o}={ }^{b} M_{c}{ }^{c} M_{o}$. Next, three waypoint frames are defined (see fig. $15 \mathrm{~b}$ ): 1)Pre-manipulation, 2) Object and 3) Manipulation. To reach each waypoint (frame), the system calculates the Cartesian distance from the end-effector to the waypoint, and drives the arm in the Cartesian space. Since this experiment was performed using the ECA CSIP Lightweight robot arm (see fig.16) which has only $4 \mathrm{DOF}$, the orientation of the waypoint is not taken into account. First the arm is driven to the pre-manipulation pose, and then to the manipulation pose, where the intervention is performed.

\subsection{Learning for Free Floating Based Manipulation on a Subsea Panel}

This is a more challenging environment where the AUV is hovering in front of the panel, compensating the environment perturbations, but has the advantage of combining the motion of the AUV and the arm to compensate for the limitations of the manipulator workspace. 


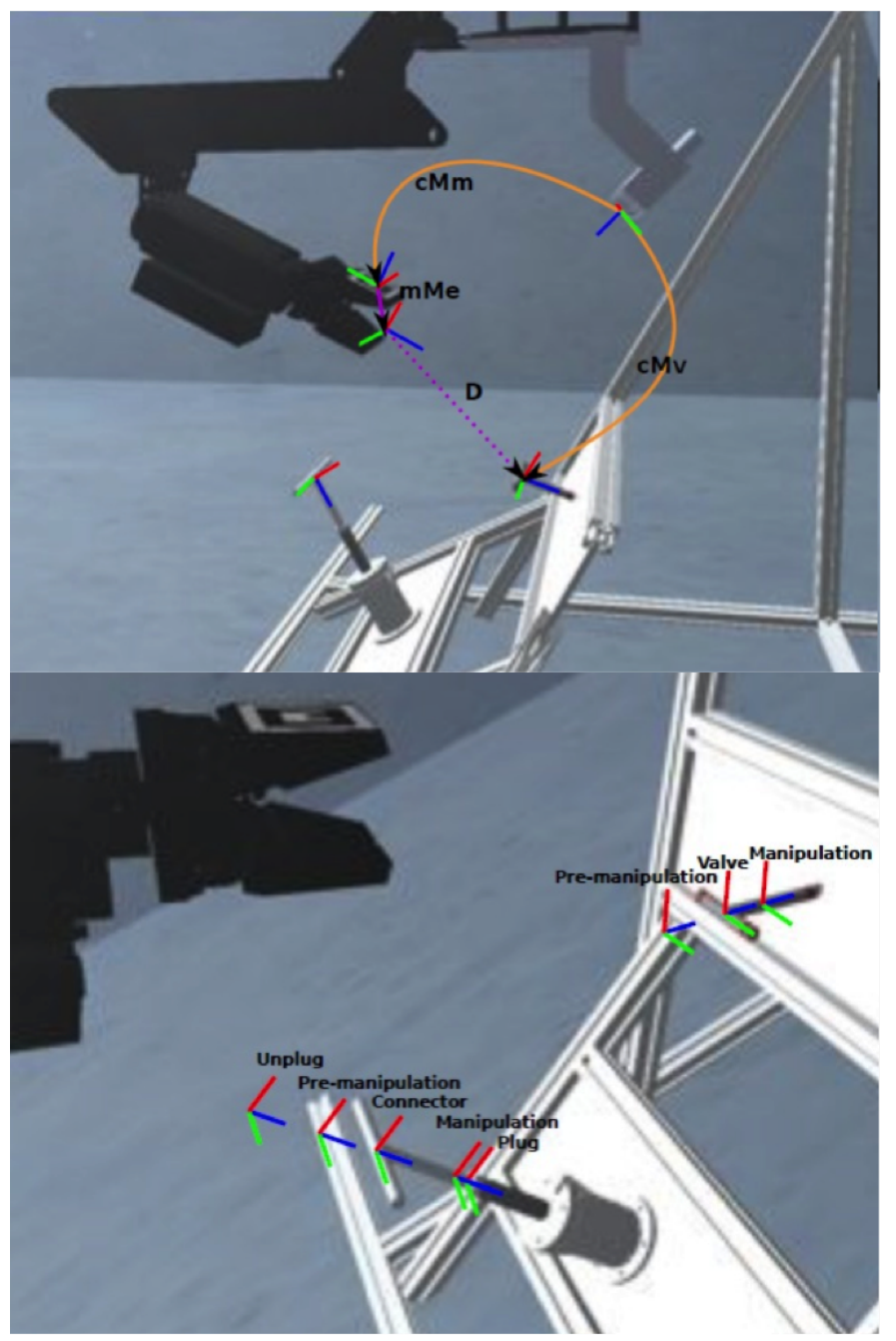

Fig. 15. top) System frames and transformations; bottom) Waypoints of the interventions.

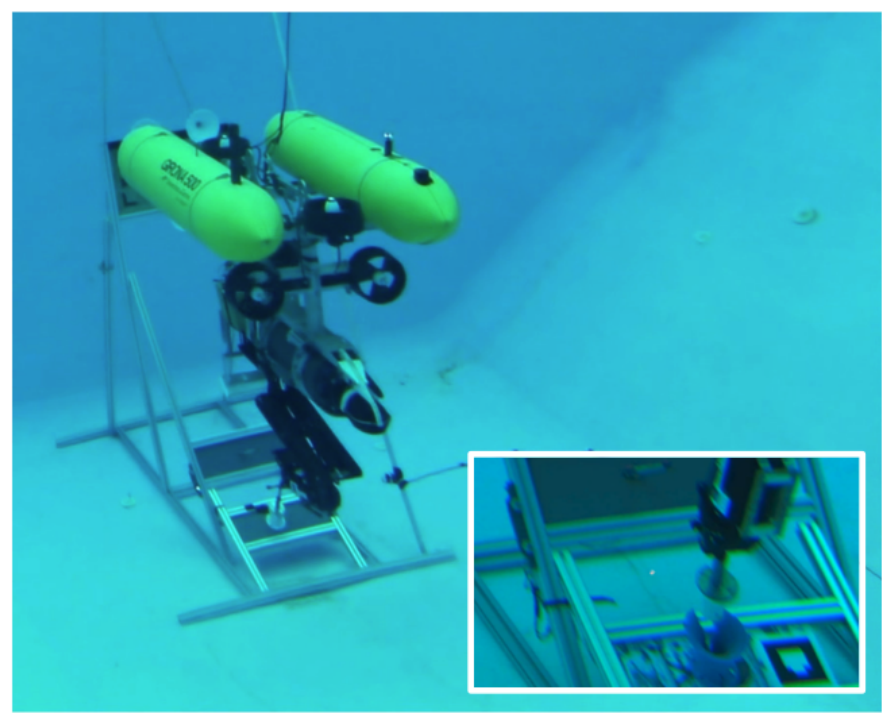

Fig. 16. GIRONA 500 in the TRITON configuration connecting a hot stab.
Problem statement Given a subsea panel equipped with T-shaped valves, the I-AUV has to be able to simultaneously control the AUV and the arm in order to perform an autonomous valve turning task in free floating configuration. A visual feature-rich textured panel is used to allow for real-time vision based localisation. The experiment has to start at a random position with the panel in the field of view so the robot may self-localise through visual servoing techniques.

Learning to Turn a Valve The strategy followed in this case (Carrera et al. (2014)) consists in transferring the knowledge of an ROV pilot to the I-AUV control system using Learning by Demonstration (LbD). In this case, the ROV pilot tele-operates the I-AUV in the water tank and gives different demonstrations. After the demonstration phase, using the LbD approach, a model of the task is learnt and the AUV is able to later reproduce the task autonomously. The LbD technique that has been used in this work is the dynamic movement primitives (DMP) algorithm. The DMP is a framework where the skill is encapsulated in a superposition of basis motion fields. This method has a compact representation and it generates motion trajectories that are robust against perturbations. The proposed method is an extension of DMP (Ijspeert et al. (2001)) (Hoffmann et al. (2009)) proposed by Kormushev (Kormushev et al. (2011)).

Fig. 17 represents the block diagram of the proposed method. During the demonstration, while the user manually performs the valve turning several times in different initial configurations, the DMP algorithm learns the end effector pose $(x, y, z, \phi, \theta, \psi)$ and the AUV pose $(x, y, z, \psi)$ simultaneously. Both poses are referenced to a frame located in the target valve. The output of the DMP algorithm is the learned model of the valve turning action. Later on, the autonomous grasping task is launched. Given the current I-AUV configuration (AUV and arm poses) and the already learned model, a new desired I-AUV configuration is abstracted from the model, and sent to the AUV and arm controllers. When the valve and the endeffector are aligned in the same position, a wrist motion command is issued and the valve is opened.

The sequence of images in the fig. 18 reflects the valve turning intervention, showing the AUV and end-effector motions. Images 1 and 2 show the approaching trajectory perpendicular to the panel while the arm is outside the camera field of view. Images 3 and 4 show the AUV in station keeping, while the manipulator approaches the valve. In image 5, the AUV and end-effector have been moved to grasp the valve. Finally, after executing the learned trajectory, the wrist motion command is launched turning the valve handle 90 degrees, as shown in image 6 .

\subsection{Object recovery}

The TRIDENT project proposed a new benchmark for the "search \& recovery" problem using a black-box mockup. Fig. 21 shows the envisioned scenario. First, the user programs a lawn-mower pattern trajectory for the I-AUV in order to survey a patch of the seafloor with $100 \%$ coverage. Next, the I-AUV is recovered and a high resolution seafloor photo-mosaic is built. Then the user identifies and selects the target and specifies the intervention task to be 


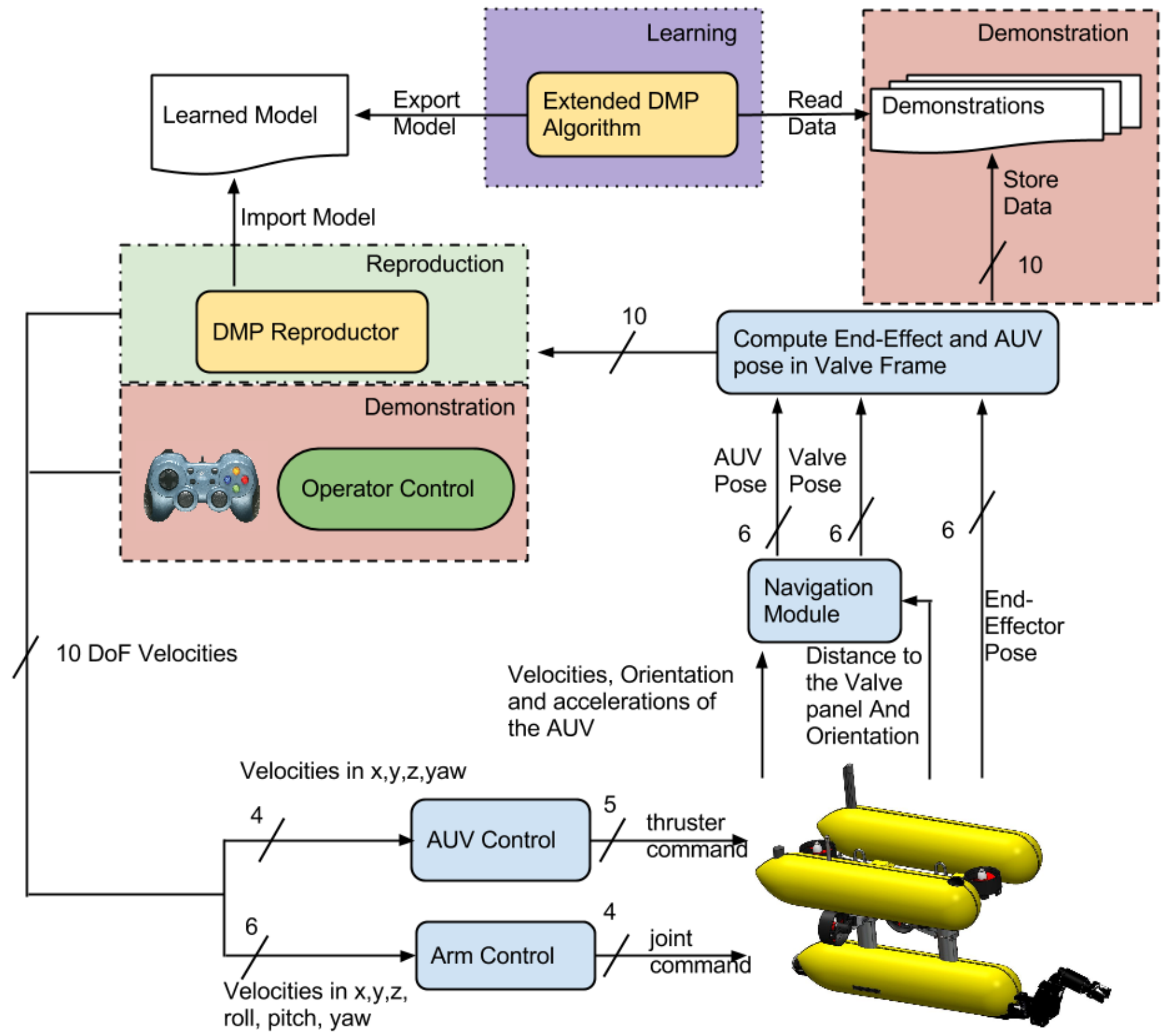

Fig. 17. Block diagram of the learning by demonstration method of autonomous valve turning.

undertaken. After, the system is launched again. The robot dives towards the target whose position is now known a priori. When the robot reaches the region of interest, it searches for it. Once the robot flies over the target and identifies it online with the bottom looking camera, it aligns itself with respect to the target, and autonomously performs the grasping operation. This benchmark has been demonstrated in different experimental conditions: 1) hooking the black-box using a 4 DOF arm in a water tank (Prats et al. (2011)), 2) hooking the black-box using a 4 DOF arm in a harbour scenario (Prats et al. (2012b)) and finally 3) grasping the black-box using a 7 DOF arm endowed with a 3 fingered hand in a harbour scenario (Sanz et al. (2012)).

Optical Survey In order to locate the object of interest (in this case, the mock-up black box), the robot is required to perform a survey of an underwater area. Apart from other sensors, the robot gathers imaging data. Using com- puter vision techniques, the surveyed area can be mapped in a common plane, which allows us to get an optical map of the seabed. Having the set of images collected by the robot, the process starts by finding a set of point features on each of them, which represent points that are surrounded by distinctive texture that should make them easily recognizable in other images. Next, the texture surrounding those points in the image is characterized using a feature descriptor. With the set of features/descriptors for each image, we search for correspondences between consecutive image pairs. To do so, we compare the descriptors of the features in each image. However, simply using the similarity between descriptors could lead to obtaining outliers, which are wrong associations between two image points that look similar but do not correspond to the same point in the sea floor. In order to avoid this, a robust model estimator is used (RANSAC), where the correspondences are considered valid if they follow the same motion model. A basic assumption behind this estimator is that 

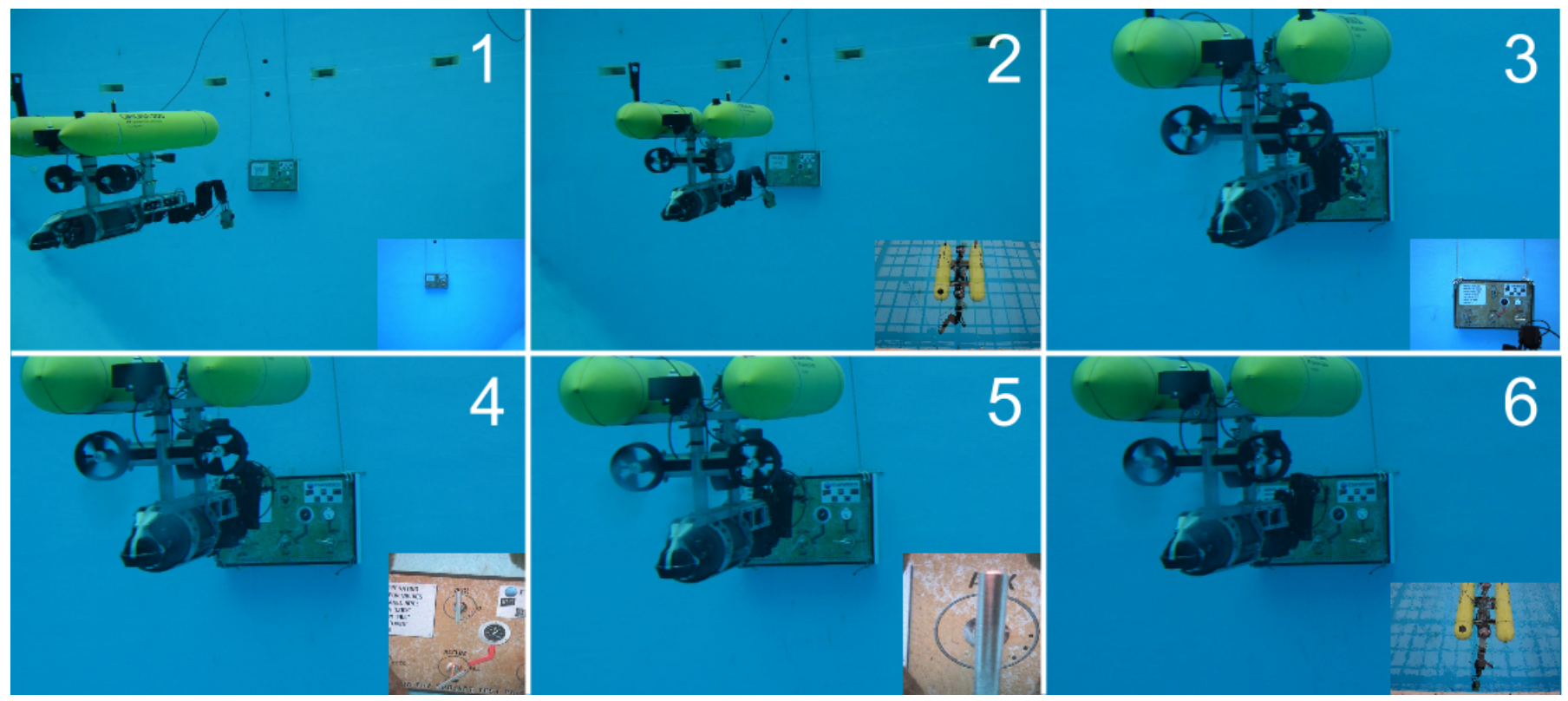

Fig. 18. Image sequence of the reproduction of a previously learned valve turning task.

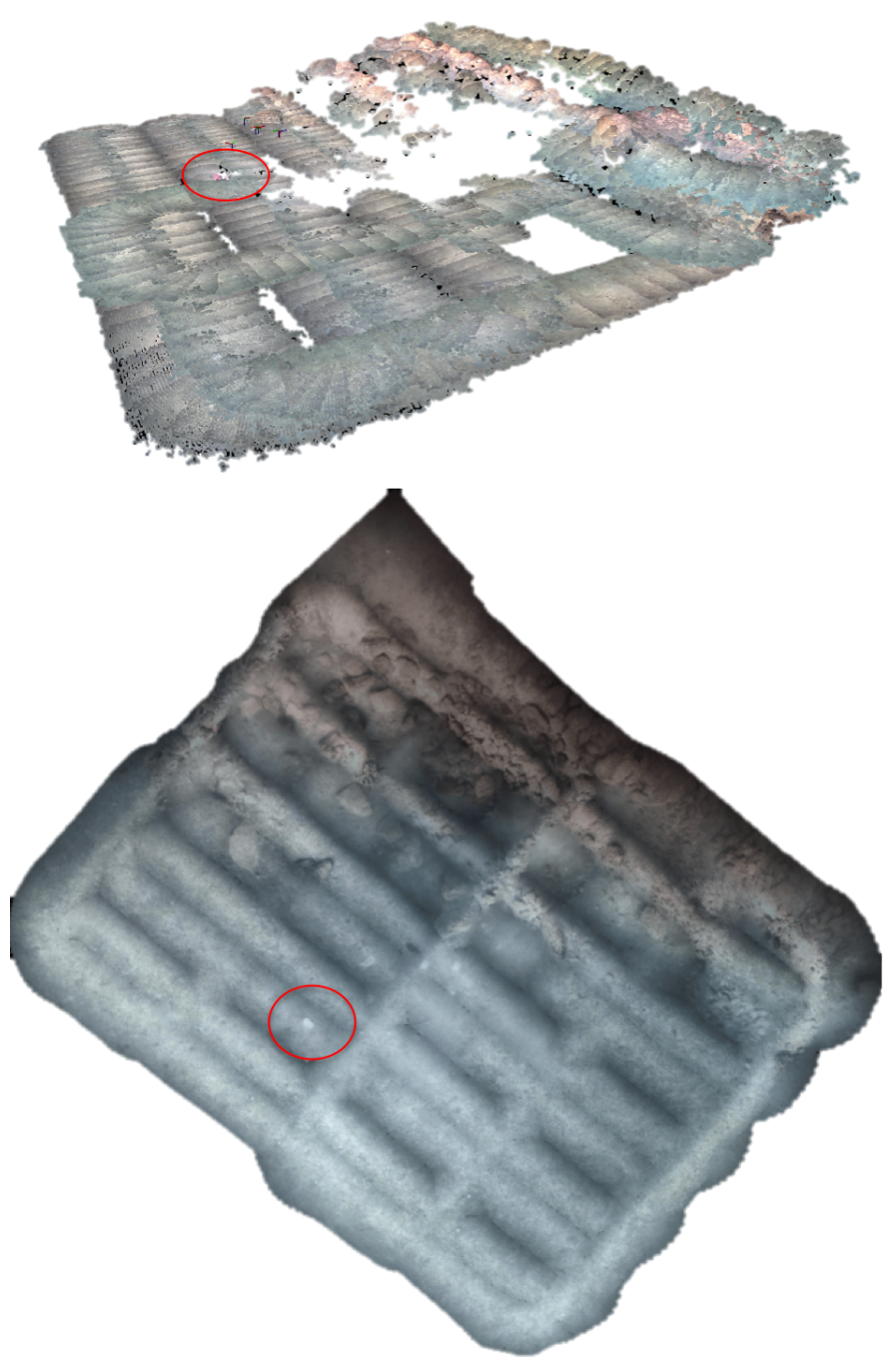

Fig. 19. $2 \mathrm{D}$ and $3 \mathrm{D}$ views of the surveyed area. we are looking at a rigid scene. With this step, we also recover the motion model in the form of a homography (3x3 matrix). Once we have the homographies between consecutive images, we can obtain a first approximation of the trajectory followed by the camera by concatenating them. As it happens with all localisation methods that rely on many sequential motion estimates to infer the robot position, errors are rapidly accumulated, and the map gets distorted. However, we can use this first approximation to find new image pairs that could correspond to the same area (overlapping images), and thus, could be matched together. Candidate matches between nonconsecutive images (loop-closures candidates) are found, and now this information is merged together. In order to do it, we perform a non-linear optimization that takes into account the re-projection error (differences between the location of the detected feature, and the estimated position after projecting using the homographies). Moreover, this optimization procedure allows including navigation data provided by other sensors of the robot. Since this navigation data is georeferenced, the resulting mosaic will also be georeferenced. Having a georeferenced mosaic, we will be able to inspect the mosaic offline, locate the object of interest we are looking for, and program the robot to go back to this position to perform the intervention task. Fig. 19 shows the 2D photo-mosaic of the field experiment performed in the Soller Harbour in Mallorca (Spain). It is also shown a $3 \mathrm{D}$ map built by compounding the disparity maps, captured by the down-looking stereo camera, with the robot poses along the trajectory. The black box is marked in both cases.

Target Detection and Tracking Because the target identification and localisation process is used as feedback to the Free Floating Controller, the detection (or tracking) frequency must be high enough to enable the controller to react on externally imposed vehicle movements. We therefore require the detector to work at a minimum frequency of $5 \mathrm{~Hz}$. Different approaches were developed based on the scene conditions and the type of sensors available. First 
versions were based on colour and shape of the target; later ones were based on image features and allowed to fully recover $3 \mathrm{D}$ information of the vehicle-to-target relative position. In the version used for the TRIDENT final experiments, the user only had to mark the grasp points of the object in a single monocular training image. Interest point coordinates and their descriptors were extracted from the whole image and stored as an object model. During the detection stage, interest points and descriptors were extracted from the current view. Using stereo vision, the $3 \mathrm{D}$ coordinates of these interest points were computed. Correspondences from the training to the current view were established by matching the feature descriptors. Minimising the re-projection error of the matched 3D points projected on the training image, the pose of the camera while taking the training image was computed. This pose and the 2D grasp points together with the point cloud generated from the current stereo view were then used to calculate the $3 \mathrm{D}$ grasp points. This way, simple $2 \mathrm{D}$ interactions are sufficient for object detector training and grasp planning, as all 3D processing is done online during autonomous grasping.

Intervention When the vehicle is sent back to the target position, which is now known, it lands in the neighborhood of the black box. Then a local search was performed until the black box appeared in the image and was detected by the vision system. At this point, the target was detected and tracked by the above mentioned vision algorithm. The object 3D pose was used to plan the grasp. The output of the tracking system was used to control the arm end-effector towards the target. The target pose, given in camera coordinates, was transformed into end-effector coordinates through the kinematic chain composed of the camera-arm base calibration and the arm forward kinematics. This allows computing an error in the Cartesian space between the current pose of the endeffector, and the desired one, which was transformed into a control velocity reference sent as input to the free floating controller. This controller was in charge of the coordinated control of the vehicle arm system (Casalino et al. (2012)). When the end-effector reached the box, it was properly grabbed by the multisensory dexterous 3 -fingered hand, being brought to the surface.

Another approach was also developed where the object $3 \mathrm{D}$ structure is recovered by using a laser stripe emitter combined with a camera (Prats et al. (2012a)). The laser emitter is mounted on the robot arm that is in charge of performing the scanning motion from a mobile platform. For this, laser peak detection and template tracking algorithms are combined. A 3D point cloud is generated after the scanning process, and filtered with a grasp region indicated by an operator on a $2 \mathrm{D}$ view of the target object. This allows to reduce the number of $3 \mathrm{D}$ points and to delimit the grasping area. After that, a grasp planner computes a suitable grasp on the reduced point cloud, and an inverse kinematic solver computes a suitable vehicle-arm pose for reaching the object at the desired grasp pose. The mission scenario is illustrated in figure 23. An underwater robotic arm (in this case the CSIP Light-weight ARM 5E (Fernandez et al. (2013))) is attached to an AUV, although at a preliminary step, for the experiments described here a floating base was used (see figure 23, right). An underwater camera (in our case a Bowtech 550C-AL) is placed near the base of the arm and facing downwards, i.e. looking towards the ground. A laser stripe emitter (in our case the Tritech SeaStripe) is assembled on the forearm of the manipulator, and emits a laser stripe visible in the camera field of view (depending on the arm joint configuration). It is assumed that the AUV has reached a pose where the target of interest is visible in the image, and that this pose is known with respect to a global fixed frame $(W)$. Figure 25 shows the robot arm and the laser plane during the scanning process and figure 24 reports the resulting point cloud. Finally, the grasping sequence is shown in figure 22 .

\section{LESSONS LEARNED}

Although first articles about intervention systems date to the mid 90's, experimental results in realistic scenarios did not arrive until the first decade of the $21^{\text {th }}$ century. Moreover, still now, field results are scarce in this area of research. This is mainly due to the complexity of the mechatronics of the involved systems. Although it is possible nowadays to acquire a low cost AUV, or several ROVs, endowed with arms, there are no I-AUVs available in the market yet. It has to be designed and built. Prior to the GIRONA 500 I-AUV only heavy I-AUVs like SAUVIM (6 Ton) or ALIVE (3.5 Ton) existed. With our design, we demonstrated light intervention capabilities with one order of magnitude less in weight $(<200 \mathrm{~kg})$. There have been two key factors which allowed us to succeed. First, the three hull design has proven to be a very stable platform, in particular when the arm is located in the bottom configuration to manipulate objects on the seafloor (fig. 11-a and b). Second, the recent appearance of low-cost and lowweight electric-arms in the market, allowed the adaption of already existing mechatronics instead of going through the slow process of designing and building a complete new arm. The ECA/CSIP arm 5E with only $27 \mathrm{~kg}$ in air (18.5 kg in water) was chosen as the candidate arm for our small I-AUV. Nevertheless, instead of just acquiring an off-the-shelf system, we went through a customisation process with the company engineers. The aim was to reduce as much weight as possible, trying to make the arm neutrally buoyant component by component. The result was the ECA/CSIP Light Weight 5E/arm (Fernandez et al. (2013)), with only $17.2 \mathrm{~kg}$ ( $3.8 \mathrm{~kg}$ in water), being currently commercialised by the company. Later on, the company built a second model, the ECA/CSIP 5E micro, even lighter with only $10 \mathrm{~kg}$ in air $(2.2 \mathrm{~kg}$ in water). The major constrain of these arms is the reduced number of available DOFs, which may be partially alleviated through the additional DOFs provided by the vehicle, achieving a redundant system with 8 DOFs (surge, sway, heave and yaw in the vehicle and base, shoulder, elbow and wrist in the arm). The second constrain of these arms is due to its simple mechatronics and poor instrumentation. In the TRIDENT project, GRAALTECH company was responsible for the design and development of a new lightweight dexterous arm. With 7 DOF, the arm weights $28 \mathrm{~kg}$ (13 $\mathrm{kg}$ in water) and was endowed with a 3 fingered hand weighting $4.5 \mathrm{~kg}$ ( $1 \mathrm{~kg}$ in water). At the cost of a higher weight and mobile mass, the use of harmonic drives and an advanced arm instrumentation, provides an accurate 


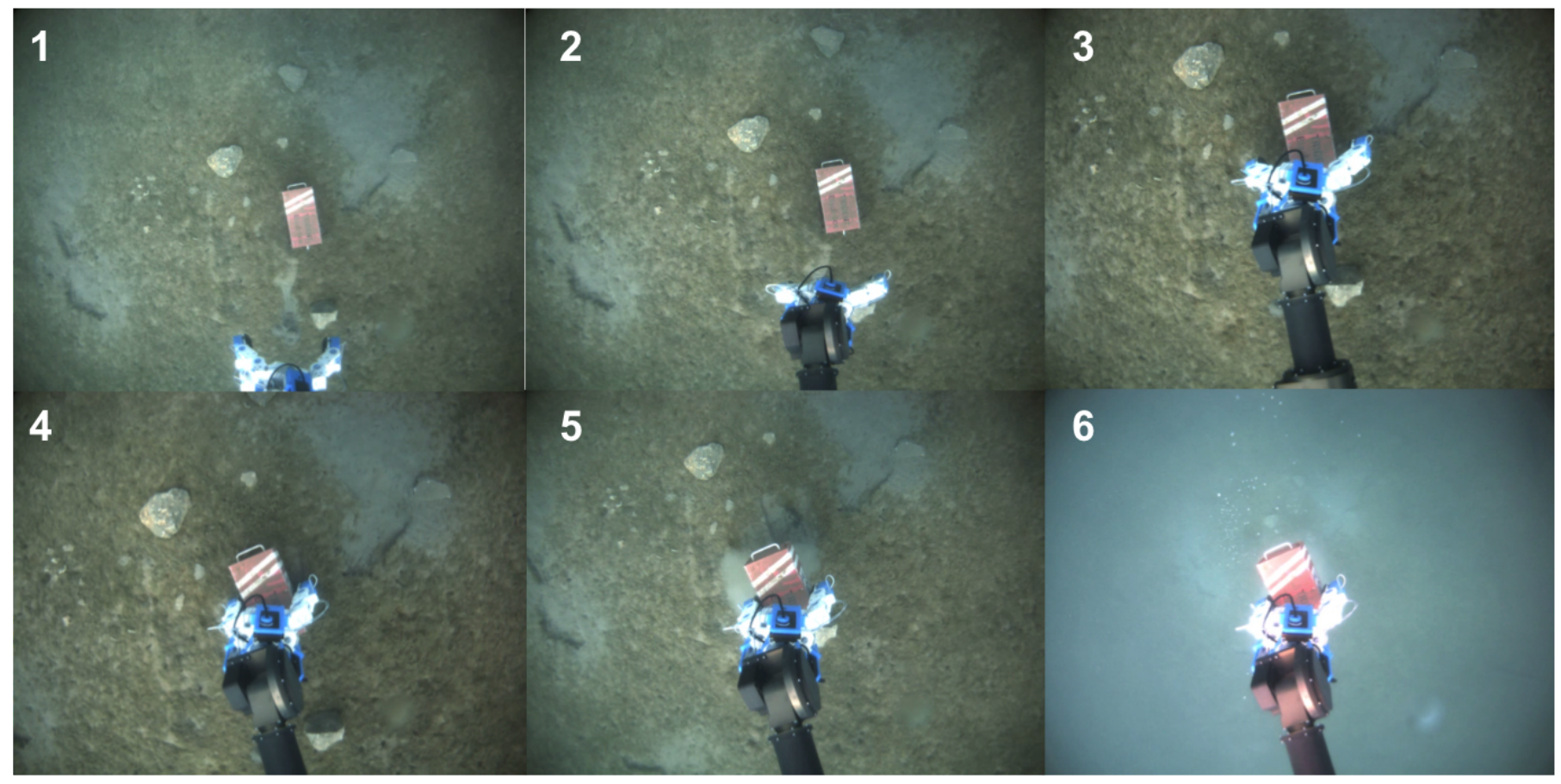

Fig. 20. Autonomous object grasping.
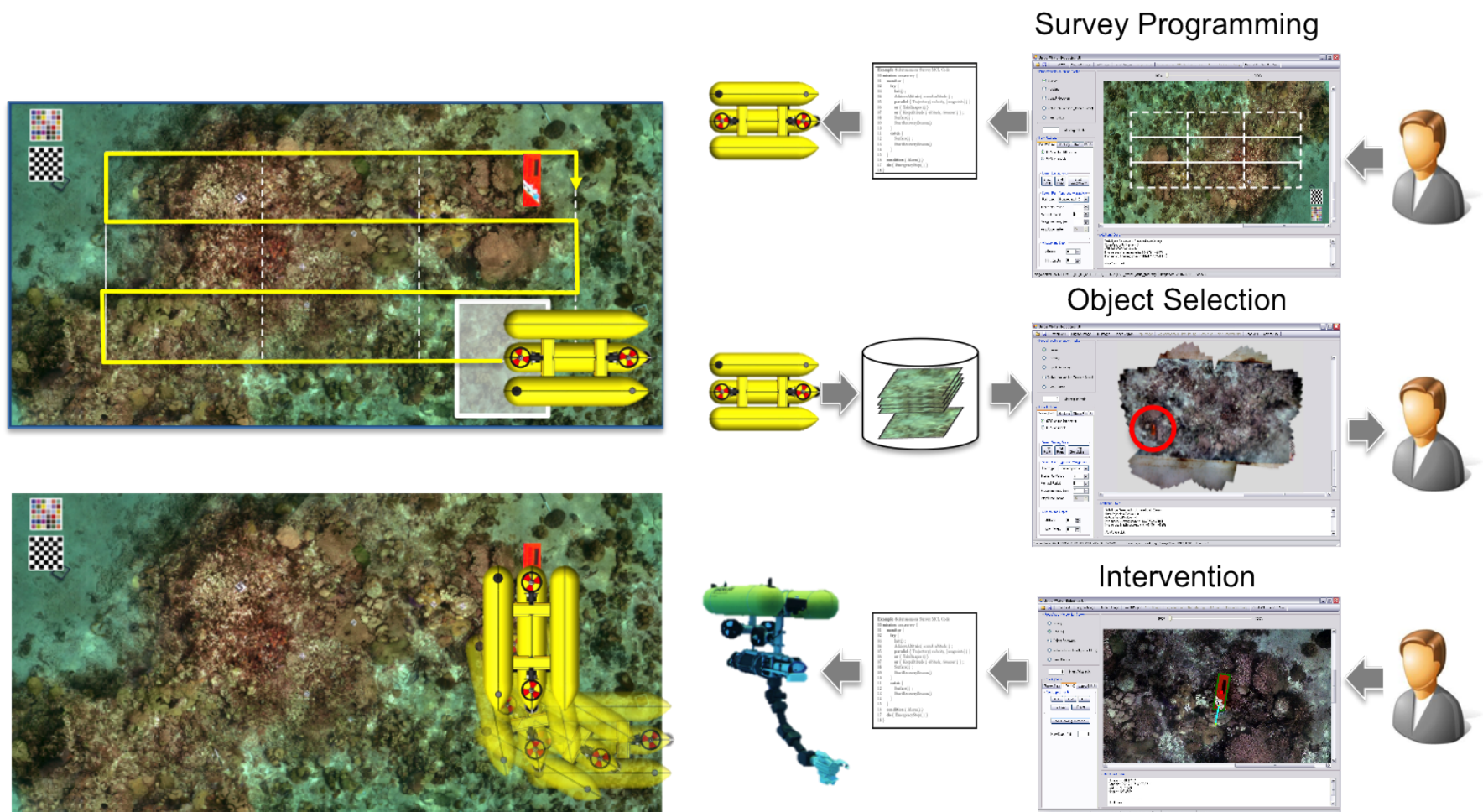

Object Selection
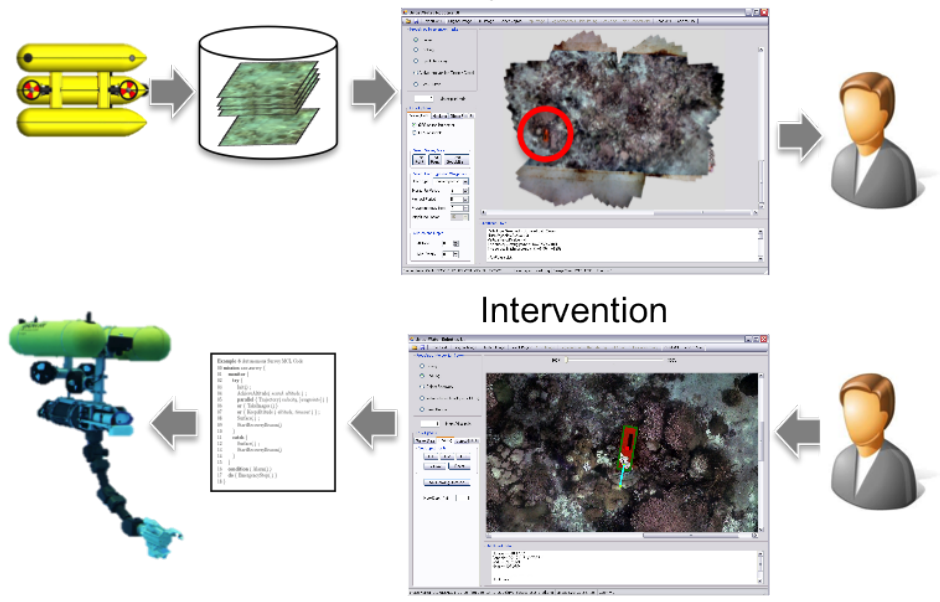

Fig. 21. Multipurpose Multisensory Based Intervention Concept.

smooth motion in the order of magnitude of standard industrial robots, enabling dexterity. Moreover, the arm redundancy together with the vehicle-arm cooperative control (Casalino et al. (2012)) allowed the system to perform the object grasping described in section 6.3 even in the presence of the failure of one of the DOF of the arm.

Another key factor to achieve experimental results quickly, has been the systems integration (Ribas et al. (2015)).
From the hardware point of view, a clear definition of the payload area and a rich signal interface, has allowed for an easy integration of the three manipulators shown in fig.11. To promote modularity and easy integration, often the payloads have included their own computer system connected to the vehicle computer through an Ethernet. From the software point of view, the adoption of the ROS middleware (Quigley et al. (2009)) has proven to be of 


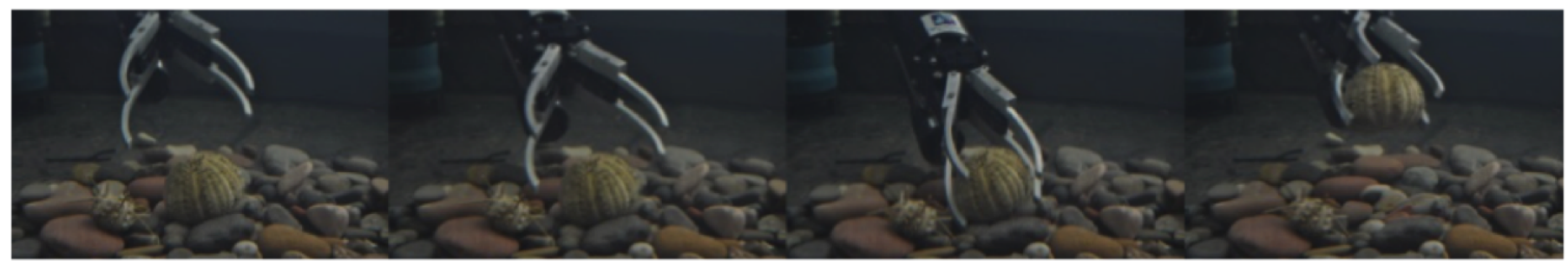

Fig. 22. Autonomous execution of the grasps by the robot.

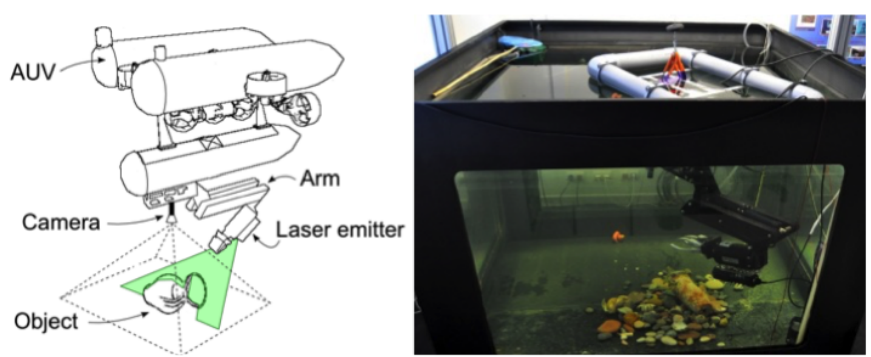

Fig. 23. The laser stripe emitter is mounted on a manipulator forearm that performs a scan of the object to be grasped. The manipulator is the Lightweight ARM 5E. (Left) ENVISIONED CONCEPT.The AUV design of this picture corresponds to the future integration with the Girona 500 vehicle (Right) REAL TESTBED used for the experiments. The arm is attached to a floating platform (4 DOF) that is placed in the water.

vital importance. It has allowed to design and implement the AUV NGC (Navigation, Guidance and Control) nodes, the computer vision nodes and the arm control nodes independently and later on integrate them in a very straight forward manner.

Another important aspect, which played an important role, was the adoption of a clear experimental methodology based on three sequential steps: 1) Hardware in the loop simulation (HIL); 2) water tank testing and 3) Sea trials. Using the UWSim (Prats et al. (2012c)) it was possible to develop the software components without actual access to the real mechatronics. In multiple partner projects, it allows to conduct the first software integration without the needs of mobilising human resources, and hence, decreasing costs. Next, mechatronics from different partners (arm, vision system, etc.) were integrated to complete the I-AUV at CIRS (Centre d'Investigaci Submarina) at UdG installations, where preliminary water tank tests were carried out. This ensured mechatronics worked properly, so it did the software integration. Finally, when everything run flawlessly, sea trials, commonly in a harbour environment were carried out to test the system in a realistic environment, validating the proposed method.

\section{CONCLUSION}

This paper has reported the main advances in autonomous underwater intervention during the last 20 years. The most relevant projects in the area have been discussed, highlighting their principal contributions. Then, the GIRONA 500 lightweight I-AUV being used in 4 different projects about underwater intervention has been described. Three different scenarios with experimental results have been
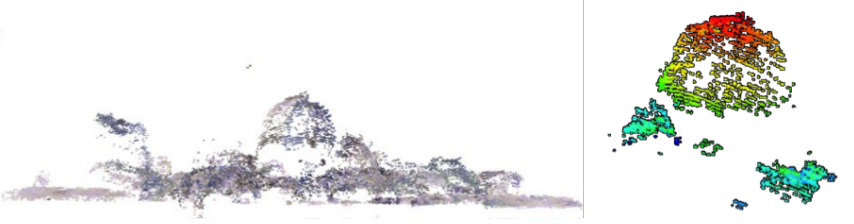

Fig. 24. laser scan of a sea urchin (left), and the camera view and user grasp specification (right). The laser stripe is detected on the image and used for the reconstruction of the $3 \mathrm{D}$ points..

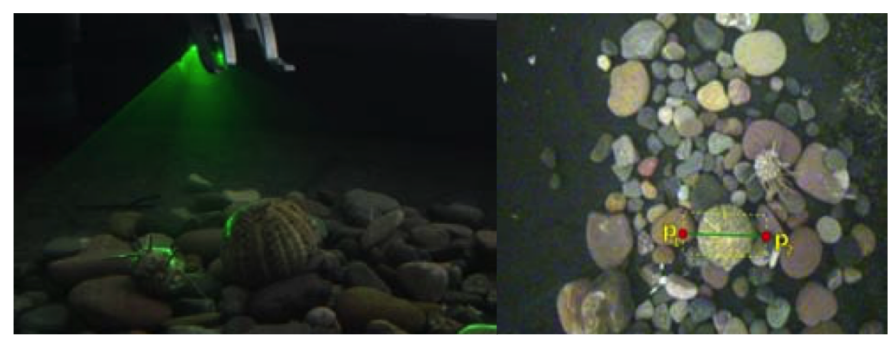

Fig. 25. Associated results to the sea urchin. (Left) The reconstructed point clouds seen from the side (Right) The original point cloud is intersected with the grasp area specified by the user, and only the points inside that area are kept. Then, outliers are removed and the point cloud is down sampled. Red pixels indicate more proximity to the camera.

presented: 1) Docking and fixed-base Manipulation, 2) Learning by demonstration for free-floating manipulation and 3) Multipurpose manipulation for object recovery. From this experience, the lessons learned have been discussed.

\section{AUTHORS}

Dr. Pere Ridao is Associate Professor and the director of Computer Vision and Robotics Research Institute at the University of Girona (Spain) and the head of the 'Girona Underwater Vision and Robotics' lab. His research activity is mainly focused on design of intelligent inspection and intervention AUVs, in research topics such as control architectures, Simultaneous Localization and Mapping (SLAM) and autonomous underwater intervention.

Dr. Marc Carreras is Associate Professor in the Computer Vision and Robotics Research Institute at the University of Girona (Spain) and member of the 'Girona Underwater Vision and Robotics' lab. His research activity is mainly focused on design of intelligent inspection and intervention AUVs, in research topics such as control architectures, robot learning and motion planning. 
Dr. David Ribas is a researcher in the Computer Vision and Robotics Research Institute at the University of Girona, and a member of the Girona Underwater Vision and Robotics lab. His research interests are focused in the development of AUVs and more particularly to the autonomous navigation problem using Simultaneous Localization and Mapping (SLAM) techniques.

Dr. Pedro J. Sanz is Full Professor at the Computer Science and Engineering Department of the Jaume I University, and the Head of the Interactive and Robotic Systems Lab. His current research interests include multisensory based grasping and dexterous manipulation, telerobotics and human?robot interaction, all of them applied to real scenarios, including assistive and underwater robotics.

Dr. Gabriel Oliver is Associate Professor at the Mathematicas and Computer Science Department of the University of the Balearic Islands, and leader of the Systems, Robotic and Vision group. His major research interests include vision-based robotic applications and architectures for navigation, guidance and control of underwater vehicles.

\section{REFERENCES}

ACSA-ALCEN (2013). Black box search and relocation. URL http://www.acsa-alcen.com/search-rescue/ black-boxes-search-relocation.

Bay, H., Ess, A., Tuytelaars, T., and Van Gool, L. (2008). Speeded-Up Robust Features (SURF). Comput. Vis. Image Underst., 110(3), 346359. doi: 10.1016/j.cviu.2007.09.014.

Bingham, B., Foley, B., Singh, H., Camilli, R., Delaporta, K., Eustice, R., Mallios, A., Mindell, D., Roman, C., and Sakellariou, D. (2010). Robotic tools for deep water archaeology: Surveying an ancient shipwreck with an autonomous underwater vehicle. J. Field Robotics, 27(6), 702-717.

Calonder, M., Lepetit, V., Strecha, C., and Fua, P. (2010). BRIEF: binary robust independent elementary features. In Proceedings of the 11th European conference on Computer vision: Part IV, ECCV'10, 778792. SpringerVerlag, Berlin, Heidelberg.

Carrera, A., Palomeras, N., Ribas, D., Kormushev, P., and Carreras, M. (2014). An intervention-auv learns how to perform an underwater valve turning. In OCEANS 2014 - TAIPEI, 1-7. doi:10.1109/OCEANSTAIPEI.2014.6964483.

Casalino, G., Angeletti, D., Cannata, G., and Marani, G. (2002). The functional and algorithmic design of amadeus multirobot workcell. In S.K. Choi and J. Yuh (eds.), Underwater Vehicle Technology, volume 12.

Casalino, G., Zereik, E., Simetti, E., Torelli, S., Sperinde, A., and Turetta, A. (2012). Agility for underwater floating manipulation: Task amp; subsystem priority based control strategy. In Intelligent Robots and Systems (IROS), 2012 IEEE/RSJ International Conference on, 1772-1779. doi:10.1109/IROS.2012.6386127.

Choi, S., Takashige, G., and Yuh, J. (1994). Experimental study on an underwater robotic vehicle: ODIN. AUV'94. Proceedings of the 1994 Symposium Autonomous Underwater Vehicle Technology, 79-84.

Cieslak, P., Ridao, P., and Giergiel, M. (2015). Autonomous underwater panel operation by girona 500 uvms: A practical approach to autonomous underwater manipulation. In Robotics and Automation (ICRA), 2015 IEEE International Conference on, 529-536. doi: 10.1109/ICRA.2015.7139230.

Drogou07 (2007). Recommendations for marine science observatory intervention. ESONET: European Seas Observatory Network. Project Deliverable D2\%.

Escartín, J., García, R., Delaunoy, O., Ferrer, J., Gracias, N., Elibol, A., Cufi, X., Neumann, L., Fornari, D.J., Humphris, S.E., and Renard, J. (2008). Globally aligned photomosaic of the Lucky Strike hydrothermal vent field (Mid-Atlantic Ridge, 3718.5N): Release of georeferenced data, mosaic construction, and viewing software. Geochem. Geophys. Geosyst., 9(12).

Eustice, R., Singh, H., and Leonard, J.J. (2006). Exactly Sparse Delayed-State Filters for View-Based SLAM. IEEE Transactions on Robotics, 22(6), 1100-1114.

Evans, J., Keller, K., Smith, J., Marty, P., and Rigaud, O. (2001). Docking techniques and evaluation trials of the SWIMMER AUV: an autonomous deployment AUV for work-class ROVs. OCEANS, 2001. MTS/IEEE Conference and Exhibition, 1, 520-528.

Evans, J., Redmond, P., Plakas, C., Hamilton, K., and Lane, D. (2003). Autonomous docking for InterventionAUVs using sonar and video-based real-time 3D pose estimation. OCEANS 2003, 4, 2201-2210.

Farr, N., Bowen, A., Ware, J., Pontbriand, C., and Tivey, M. (2010). An integrated, underwater optical /acoustic communications system. In OCEANS 2010 IEEE Sydney, 1-6. doi:10.1109/OCEANSSYD.2010.5603510.

Fernandez, J., Prats, M., Sanz, P., Garcia, J., Marin, R., Robinson, M., Ribas, D., and Ridao, P. (2013). Grasping for th seabed: Developing a new underwater robot arm for shallow-water intervention. Robotics Automation Magazine, IEEE, $\mathrm{PP}(99), 1-1$. doi: 10.1109/MRA.2013.2248307.

Ferrer, J., Elibol, A., Delaunoy, O., Gracias, N., and Garcia, R. (2007). Large-Area Photo-Mosaics Using Global Alignment and Navigation Data. MTS/IEEE OCEANS, Vancouver.

Fletcher, B., Young, C., Buescher, J., Whitcomb, L., Bowen, A., McCabe, R., and Yoerger, D. (2008). Proof of concept demonstration of the Hybrid Remotely Operated Vehicle (HROV) light fiber tether system. OCEANS 2008, 1-6.

Gilmour, B. (2012). Field resident AUV systems: Chevrons long-term goal for Autonomous Underwater Vehicle (AUV) Development. Oceanic Engineering Society IEEE AUV 2012.

Hoffmann, H., Pastor, P., Park, D.H., and Schaal, S. (2009). Biologically-inspired dynamical systems for movement generation: Automatic real-time goal adaptation and obstacle avoidance. In ICRA, 25872592. IEEE.

Ijspeert, A., Nakanishi, J., and Schaal, S. (2001). Trajectory formation for imitation with nonlinear dynamical systems. In Intelligent Robots and Systems, 2001. Proceedings. 2001 IEEE/RSJ International Conference on, volume 2, 752757 vol.2. doi:10.1109/IROS.2001.976259.

Inglis, G., Smart, C., Vaughn, I., and Roman, C. (2012). A pipeline for structured light bathymetric mapping. IEEE/RSJ International Conference on Intelligent Robots and Systems, (Figure 1), 4425-4432. 
Johnson-Roberson, M., Pizarro, O., Williams, S.B., and Mahon, I. (2010). Generation and Visualization of Large-Scale Three-Dimensional Reconstructions from Underwater Robotic Surveys. Journal of Field Robotics, 27(1), 21-51. doi:10.1002/rob.

Kaiser, C., A., B., Camilli, R., Yoerger, D., and Jakuba, M. Farr, N. (2013). Hybrid auv inspection, monitoring, and intervention of seafloor and sub-seafloor pipelines. URL http://www. whoi.edu/page.do?pid=119416 \& tid=3622 \& cid=163610.

Kato, H. and Billinghurst, M. (1999). Marker tracking and hmd calibration for a video-based augmented reality conferencing system. In Augmented Reality, 1999. (IWAR '99) Proceedings. 2nd IEEE and ACM International Workshop on, 85-94. doi: 10.1109/IWAR.1999.803809.

Kormushev, P., Calinon, S., and Caldwell, D.G. (2011). Imitation Learning of Positional and Force Skills Demonstrated via Kinesthetic Teaching and Haptic Input. Advanced Robotics, 25(5), 581603.

Lane, D.M., Maurelli, F., Larkworthy, T., Caldwell, D., Salvi, J., Fox, M., and Kyriakopoulos, K. (2012). Pandora: Persistent autonomy through learning, adaptation, observation and re-planning. In Proceedings of IFAC NGCUV 2012 - Navigation, Guidance and Control of Underwater Vehicles.

Lane, D., O'Brien, D.J., Pickett, M., Davies, J., Robinson, G., Jones, D., Scott, E., Casalino, G., Bartolini, G., Cannata, G., Ferrara, A., angeletti, D., Veruggio, G., Bono, R., Virgili, P., Canals, M., Pallas, R., Garcia, E., and Smith, C. (1997). AMADEUS-Advanced Manipulation for Deep Underwater Sampling. IEEE Robotics and Automation Magazine, 4(4), 34-45.

Lowe, D.G. (2004). Distinctive Image Features from ScaleInvariant Keypoints. Int. J. Comput. Vision, 60(2), 91110. doi:10.1023/B:VISI.0000029664.99615.94.

Marani, G., Choi, S., and Yuh, J. (2009). Underwater Autonomous Manipulation for Intervention Missions AUVs. Ocean Engineering. Special Issue: AUV, 36(1), $15-23$.

Nicosevici, T., Gracias, N., Negahdaripour, S., and Garcia, R. (2009). Efficient three-dimensional Scene Modeling and Mosaicing. Journal of Field Robotics, 26(10), 759788.

Paduan, J.B., Caress, D.W., Clague, D.A., Paull, C.K., and Thomas, H. (2009). High-resolution mapping of mass wasting, tectonic, and volcanic hazards using the MBARI Mapping AUV. International Conference on seafloor mapping for geohazard assessmen, 7, 181-186.

Palomeras, N., Nagappa, S., Ribas, D., Gracias, N., and Carreras, M. (2013). Vision-based localization and mapping system for AUV intervention. In OCEANS'13 $M T S / I E E E$.

Palomeras, N., Penalver, A., Massot-Campos, M., Vallicrosa, G., Negre, P., Fernandez, J., Ridao, P., Sanz, P., Oliver-Codina, G., and Palomer, A. (2014). Iauv docking and intervention in a subsea panel. In Intelligent Robots and Systems (IROS 2014), 2014 IEEE/RSJ International Conference on, 2279-2285. doi:10.1109/IROS.2014.6942870.

Pizarro, O., Eustice, R., and Singh, H. (2009). Large area $3-\mathrm{D}$ reconstructions from underwater optical surveys. IEEE Journal of Oceanic Engineering, 34(2), 150-169.
Prats, M., Fernandez, J., and Sanz, P. (2012a). Combining template tracking and laser peak detection for $3 \mathrm{~d}$ reconstruction and grasping in underwater environments. In Intelligent Robots and Systems (IROS), 2012 IEEE/RSJ International Conference on, 106-112. doi: 10.1109/IROS.2012.6385792.

Prats, M., García, J., Fernandez, J., Marín, R., and Sanz, P. (2011). Advances in the specification and execution of underwater autonomous manipulation tasks. IEEE Oceans 2011, 10361-10366.

Prats, M., García, J., Wirth, S., Ribas, D., Sanz, P., Ridao, P., Gracias, N., and Oliver, G. (2012b). Multipurpose autonomous underwater intervention: A systems integration perspective. Control \& Automation (MED), 2012 20th Mediterranean Conference on, 1379-1384.

Prats, M., Perez, J., Fernandez, J., and Sanz, P. (2012c). An open source tool for simulation and supervision of underwater intervention missions. In Intelligent Robots and Systems (IROS), 2012 IEEE/RSJ International Conference on, 2577-2582. doi:10.1109/IROS.2012.6385788.

Prats, M., Ribas, D., Palomeras, N., Garcia, J., Nannen, V., Wirth, S., Fernandez, J.J., Beltran, J., Campos, R., Ridao, P., Sanz, P., Oliver, G., Carreras, M., Gracias, N., Marin, R., and Ortiz, A. (2012d). Reconfigurable auv for intervention missions: a case study on underwater object recovery. Intelligent Service Robotics, 5(1), 19-31. doi:10.1007/s11370-011-0101-z. URL http: //dx.doi.org/10.1007/s11370-011-0101-z.

Quigley, M., Conley, K., Gerkey, B.P., Faust, J., Foote, T., Leibs, J., Wheeler, R., and Ng, A.Y. (2009). Ros: an open-source robot operating system. In International Conference on Robotics and Automation (ICRA), 2009 Workshop on Open Source Software.

Ribas, D., Palomeras, N., Ridao, P., Carreras, M., and Mallios, A. (2012). Girona 500 auv: From survey to intervention. Mechatronics, IEEE/ASME Transactions on, 17(1), 46-53. doi:10.1109/TMECH.2011.2174065.

Ribas, D., Ridao, P., Turetta, A., Melchiorri, C., Palli, G., Fernandez, J., and Sanz, P. (2015). I-auv mechatronics integration for the trident $\mathrm{fp} 7$ project. Mechatronics, IEEE/ASME Transactions on, $\mathrm{PP}(99), 1-10$. doi: 10.1109/TMECH.2015.2395413.

Richmond, K. and Rock, S. (2007). An Operational RealTime, Large-Scale Visual Mosaicking And Navigation System. Sea Technology, 48, 10-13.

Ridao, P., Carreras, M., Ribas, D., and García, R. (2010). Visual inspection of hydroelectric dams using an autonomous underwater vehicle. J. Field Robotics, 27(6), $759-778$.

Rigaud, V., Coste-Maniere, E., Aldon, M., Probert, P., Perrier, M., Rives, P., Simon, D., Lang, D., Kiener, J., Casal, A., Amar, J., Dauchez, P., and Chantler, M. (1998). UNION: underwater intelligent operation and navigation. Robotics \& Automation Magazine, IEEE, $5(1), 25-35$.

Rosten, E. and Drummond, T. (2006). Machine learning for high-speed corner detection. In In European Conference on Computer Vision, 430443.

Rublee, E., Rabaud, V., Konolige, K., and Bradski, G. (2011). ORB: An efficient alternative to SIFT or SURF. In Computer Vision (ICCV), 2011 IEEE International Conference on, 25642571. doi: 
10.1109/ICCV.2011.6126544.

Sanz, P., Marín, R., Sales, J., Oliver, G., and Ridao, P. (2012). Recent advances in underwater robotics for intervention missions. Soller harbor experiments. Low Cost Books, Spain.

Sigh, H., Howland, J., and Pizarro, O. (2004). Advances in large-area photomosaicking underwater. IEEE Journal of Oceanic Engineering, 29, 872-886.

SPLASHCOS (2013). Submerged prehistoric archaeology and landscapes of the continental shelf. URL http://www.splashcos.org/.

Vallicrosa, G., Ridao, P., Ribas, D., and Palomer, A. (2014). Active range-only beacon localization for auv homing. In Intelligent Robots and Systems (IROS 2014), 2014 IEEE/RSJ International Conference on, 22862291. doi:10.1109/IROS.2014.6942871.

Wang, H.H., Rock, S.M., and Lees, M.J. (1995). Experiments in automatic retrieval of underwater objects with an AUV. OCEANS'95. MTS/IEEE. Challenges of Our Changing Global Environment. Conference Proceedings, 1, 366-373. 